\title{
Elastic-plated gravity currents
}

\author{
I. J. HEWITT ${ }^{1}$, N. J. BALMFORTH ${ }^{1}$ and J. R. DE BRUYN ${ }^{2}$ \\ ${ }^{1}$ Department of Mathematics, University of British Columbia, Vancouver, Canada \\ ${ }^{2}$ Department of Physics and Astronomy, University of Western Ontario, London, Ontario, Canada
}

(Received 5 September 2014)

\begin{abstract}
We consider a nonlinear diffusion equation describing the planar spreading of a viscous fluid injected between an elastic sheet and an underlying rigid plane. The dynamics depends sensitively on the physical conditions at the contact line where the sheet is lifted off the plane by the fluid. We explore two possibilities for these conditions (or "regularizations"): a pre-wetted film and a constant-pressure fluid lag (a gas-filled gap between the fluid edge and the contact line). For both flat and inclined planes, we compare numerical and asymptotic solutions, identifying the distinct stages of evolution and the corresponding characteristic rates of spreading.
\end{abstract}

\section{Introduction}

Many physical problems involve the spreading of a viscous fluid layer under gravity. If the layer is shallow, lubrication theory is often used to describe the fluid dynamics. This furnishes a nonlinear diffusion equation for the fluid depth, $h$, that is degenerate at the edge where $h$ goes to zero. Correctly modelling the advance of this front has obvious practical importance and has attracted much research (see $[3,6])$.

In the simplest case when gravity provides the only pressure force, the diffusion equation is second order and the advance of the front can be correctly predicted by imposing the condition $h=0$ there, the front speed then being determined by mass conservation [15]. When surface tension is introduced, the 'thin film' equation is fourth order in space and an additional condition is required to determine the contact angle $\tan ^{-1}\left(h_{x}\right)$. Unfortunately, for a moving front that angle is not, in general, constant. Moreover, there are mathematical difficulties in dealing with a vanishing diffusivity at the contact line. Consequently, for practical purposes some form of regularization is usually adopted to avoid these issues. One common approach is to add a thin pre-wetted film of fluid, thus avoiding the requirement for any boundary conditions at a genuine contact line or 'front' [24]. Unsatisfyingly, however, the solution can turn out to depend upon the pre-wetted film depth, $\delta$, and may not converge to any limit as that depth is decreased towards zero [28]. Other forms of reqularization suffer from a similar problem. Indeed, even outside the confines of the thin film approximation, full continuum theories of fluid mechanics are unable to describe the flow near the contact line without introducing molecular-scale physics $[3,6]$.

In this paper we consider spreading beneath a thin elastic sheet, when pressure forces result from bending and stretching of the sheet, as well as from gravity. This gives rise 
to a sixth-order diffusion equation, for which it is again convenient to regularize the behaviour near the front [9]. We consider two alternatives: (i) a pre-wetted film, and (ii) a fluid lag. For the latter, there is a finite fluid edge, but the sheet does not touch down on the underlying substrate there; rather, the edge lags the touchdown position, leaving a small gas-filled gap. The gas in this gap is held at a given constant pressure, $-\sigma$, corresponding to that for which the fluid vaporizes, or to some other pressure if other gasses are present (if the sheet is permeable, for instance). A similar approach is commonly used in models of fluid-driven fracture $[20,10,12]$ and is motivated by the fact that the pressure close to the advancing front becomes large and negative, causing dissolved gasses to exsolve from the fluid.

We explore the planar spreading of fluid that is supplied at a constant rate from a line source on either a flat or a sloping substrate. Using a combination of numerical computation and asymptotic analysis, we determine in detail how the two regularizations of the fluid edge impact the dynamics in the limit that the pre-wetted film depth $\delta$ becomes small, or the lag pressure $\sigma$ becomes large. Our analysis is similar to two previous studies: Flitton \& King [9] examined the spreading over a flat surface of a constant volume, and Lister, Peng \& Neufeld [22] considered the axisymmetric version of our problem.

Applications for which this study is relevant include geophysical, engineering and biological problems. The growth of magma intrusions and fluid-driven opening of fractures in the Earth's crust can be described using a model of this type [23, 4], and analogous models for turbulent fluid flow have been used to describe subglacial floods [7, 27]. Similar models have application to the manufacture of silicon wafers $[19,16]$, the development of micro-electro-mechanical systems [14], the peeling of an elastic sheet from an adhesive [5], "elastocapillarity" [1], the passage of air flow in the lungs [11], the operation of vocal cords [13], and the suppression of viscous fingering [25].

The paper is organized as follows. In section 2 the physical problem is described and we introduce the governing diffusion equation. In section 3 we consider the dynamics of the front, describing the regularizations and summarizing the effect these have on the local structure of the solutions in the limit $\delta \rightarrow 0$ or $\sigma \rightarrow \infty$. We then describe the numerical and asymptotic results for solutions on the flat, concentrating on the problem

with bending stress in section 4 and with tension in section 5 . Solutions on a slope are then considered; those with bending in section 6 , and with tension in section 7 .

\section{Model equations}

\subsection{Dimensional equations}

We consider the situation shown in figure 1: a fluid is injected at the interface between a rigid substrate and a deformable elastic sheet. The normal displacement of the sheet is $h(x, t)$, and the fluid flow in the gap is modelled using lubrication theory, for which

$$
h_{t}+J_{x}=w, \quad J=\frac{h^{3}}{12 \mu}\left(\rho g \sin \theta-p_{x}\right),
$$

where $J(x, t)$ is the fluid flux along the gap, $\rho$ and $\mu$ are the fluid's density and viscosity, $\theta$ is the angle of the underlying slope, $g$ is the gravitational acceleration, and $p(x, t)$ is 
(a)

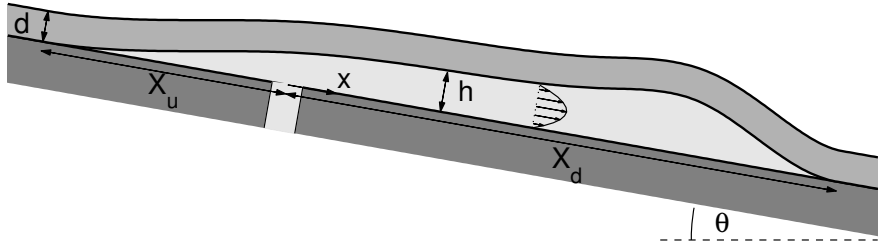

(b)

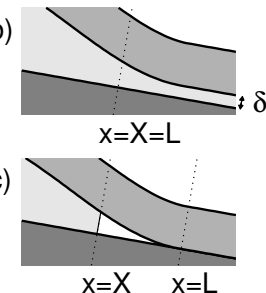

FiguRE 1. Diagram showing setup with close ups of the contact line for the two regularizations.

the pressure at the base of the fluid layer. The source term $w$ is taken to be either a point source with strength $Q$ at the origin, or is spread over a vent of finite width.

The fluid pressure is dictated by the elastic forces in the sheet and the hydrostatic pressure in the fluid. Modelling the sheet as a beam of thickness $d$, Young's modulus $E$, and Poisson ratio $\nu$, we have

$$
p=\frac{E d^{3}}{12\left(1-\nu^{2}\right)} h_{x x x x}-N h_{x x}+\rho g \cos \theta h,
$$

relative to the pressure in the absence of any deformation, which includes the uniform weight of the sheet. The tension, $N$, is given by

$$
N=E d\left(\xi_{x}+\frac{1}{2} h_{x}^{2}\right),
$$

where $\xi(x, t)$ is the in-plane displacement. In the thin layer limit, the fluid traction acting tangential to the base of the sheet is much smaller than the normal force (the lubrication pressure), and so the longitudinal force balance on the sheet demands $\partial N / \partial x=0$; i.e. $N(t)$ is uniform in space. For related reasons, the in-plane displacement $\xi$ is much smaller that the out-of-plane deflection $h$, allowing one to discard any lateral motion of the sheet in computing the fluid flux in (2.1). If there is no longitudinal displacement of the sheet for $x \geq L_{d}(t)$ and $x \leq-L_{u}(t)$, then the integral of (2.3) implies that

$$
N=\frac{E d}{L_{u}+L_{d}} \int_{-L_{u}}^{L_{d}} \frac{1}{2} h_{x}^{2} \mathrm{~d} x
$$

\subsection{Dimensionless equations}

Dimensions are removed by writing $x=\mathcal{L} \hat{x}, h=\mathcal{H} \hat{h}, t=(\mathcal{L H} / Q) \hat{t}$, and $p=\mathcal{P} \hat{p}$, where $\mathcal{L}$, $\mathcal{H}$ and $\mathcal{P}=12 \mu \mathcal{Q L} / \mathcal{H}^{3}$ are characteristic length, depth, and pressure scales. On dropping the hat decoration, the dimensionless equations become

$$
h_{t}=\left[h^{3}\left(p_{x}-\mathcal{S}\right)\right]_{x}+w,
$$

where

$$
p=\mathcal{B} h_{x x x x}-\mathcal{T} N h_{x x}+\mathcal{G} h, \quad N=\frac{1}{L_{u}+L_{d}} \int_{-L_{u}}^{L_{d}} \frac{1}{2} h_{x}^{2} \mathrm{~d} x .
$$




\begin{tabular}{lcccc}
\hline & Bending & Tension & Gravity & Slope \\
Depth & $t^{5 / 9}$ & $t^{5 / 11}$ & $t^{1 / 5}$ & $t^{0}$ \\
Length & $t^{4 / 9}$ & $t^{6 / 11}$ & $t^{4 / 5}$ & $t$ \\
\hline
\end{tabular}

Table 1. Potential similarity scalings when a particular term dominates the pressure gradient.

The source function $w(x)$ is either Dirac's delta function or is spread over a vent of finite dimensionless width, $x_{v}$; in practice, we use $\frac{3}{4} \max \left(0, x_{v}^{2}-x^{2}\right) / x_{v}^{3}$. The parameters are

$$
\mathcal{B}=\frac{E d^{3}}{12\left(1-\nu^{2}\right)} \frac{\mathcal{H}}{\mathcal{P} \mathcal{L}^{4}}, \quad \mathcal{T}=\frac{E d \mathcal{H}^{3}}{\mathcal{P} \mathcal{L}^{4}}, \quad \mathcal{G}=\frac{\rho g \cos \theta \mathcal{H}}{\mathcal{P}}, \quad \mathcal{S}=\frac{\mathcal{L}}{\mathcal{H}} \mathcal{G} \tan \theta
$$

These four parameters control the relative importance of the forces of bending and tension, and gravity acting normal to and down the slope (we refer to $\mathcal{G}$ simply as "gravity" and $\mathcal{S}$ as "slope", for short). If one of these forces dominates the others in the pressure gradient, a similarity scaling for the fluid depth and length might be expected (e.g. $[21,23])$; these scalings for a constant rate of injection are summarized in table 1 . Particular choices of the dimensional length and depth scales, $\mathcal{L}$ and $\mathcal{H}$, allow two of the parameters to be set to 1 .

\section{Contact line dynamics}

\subsection{The need for regularization}

If the fluid domain is finite, with expanding edges at $x=-X_{u}(t)$ or $X_{d}(t)$, then mass conservation requires

$$
\dot{X}=\lim _{x \rightarrow X} h^{2}\left(\mathcal{S}-p_{x}\right) \quad \text { for } X(t)=\left\{\begin{array}{l}
-X_{d} \\
X_{u}
\end{array}\right.
$$

Three further boundary conditions are required at each edge (or two if $\mathcal{B}=0$ ), and if we were to assume that the sheet meets the substrate smoothly at that point, a plausible choice is $h=h_{x}=h_{x x}=0$. However, it is in fact impossible to construct solutions to (2.5) that have a genuine edge that advances at finite speed [9]. To demonstrate this, one observes that the local behaviour as $x \rightarrow X(t)$ and $h \rightarrow 0$ is governed by

$$
-\dot{X} h_{x} \sim\left[h^{3}\left(\mathcal{B} h_{x x x x x}-\mathcal{T} N h_{x x x}+\mathcal{G} h_{x}-\mathcal{S}\right)\right]_{x} .
$$

Seeking a solution $h \sim(X-x)^{m}$ for some exponent $m$, we find the dominant balance (provided $\mathcal{B} \neq 0$ ),

$$
m \dot{X}(X-x)^{m-1} \sim \mathcal{B} m(m-1)(m-2)(m-3)(m-4)(4 m-5)(X-x)^{4 m-6} .
$$

Matching exponents requires $m=5 / 3$, but then the sign of term on the left is positive and that on the right is negative, making this balance unfeasible. A similar result holds if $\mathcal{B}=0$ [18]. We conclude that the model described by (2.5) and (2.6) on a finite domain is incomplete, and are forced to consider some form of regularization. 


\subsection{The pre-wetted film}

In this model we assume that a thin film is present everywhere and pose (2.5) on an infinite domain with $h \rightarrow \delta$ as $|x| \rightarrow \infty$. There are no longer any genuine fluid fronts, but the depth decreases sharply towards the thickness of the film at two expanding positions that can be identified as effective contact lines. For definiteness, we locate these fronts at $x=X(t)$, where $h(X, t)=2 \delta$ and $X=-X_{u}(t)$ or $X_{d}(t)$; provided $\delta \ll 1$, the precise multiple of $\delta$ chosen is of no consequence. This device further enables us to compute the tension in (2.6) if we adopt $L_{u}=X_{u}$ and $L_{d}=X_{d}$. Note that, implicitly, we therefore assume that the viscous traction in the pre-wetted film is sufficient to anchor the overlying elastic sheet in place without slipping (enforcing $\xi=0$ for $x<-X_{u}$ and $x>X_{d}$ ), even though we ignore its effect on the sheet above the main fluid current. The key point in justifying this assumption is that the pre-wetting film is introduced here as a regularization of the model, not necessarily because one is really there.

With the pre-wetted film, our strategy for numerically solving (2.5)-(2.6) is first to truncate the infinite domain and discretize $x$ with a uniform grid on a finite computational domain that is larger than the final extent of the spreading current. The tension integral is computed by quadrature and after using centred finite differences for spatial derivatives, we integrate the resulting system of ODEs in time using a standard stiff integrator. The initial condition is $h(x, 0)=\delta$, and, for purposes of illustration, we mostly include $w$ as

a line source at $x=0$. For spreading over a horizontal surface, we exploit symmetry to consider only half the domain, $0 \leq x$, imposing $h_{x}(0, t)=h_{x x x}(0, t)=0$.

\subsection{The fluid lag}

In this model the sheet loses contact with the fluid at $x=X(t)=-X_{u}(t)$ and $X_{d}(t)$, and then touches down on the substrate at $x=L(t)=-L_{u}(t)$ and $L_{d}(t)$. The regions $-L_{u}<x<-X_{u}$ and $X_{d}<x<L_{d}$ are filled with gas at pressure $-\sigma$. Over these 'lags', $h$ represents the deflection of the sheet, and the constant pressure requires (cf. (2.6)),

$$
\mathcal{B} h_{x x x x}-\mathcal{T} N h_{x x}=-\sigma .
$$

At $x=L$, we apply the contact conditions $h=h_{x}=h_{x x}=0$, and we require continuity of $h$ and its first four derivatives at the fluid fronts, $x=X$. The condition $h_{x x}=0$ assumes that there is no adhesion of the sheet to the substrate. Note that, given the hydrostatic contribution to the pressure, $p-\mathcal{G} h=-\sigma$ at the top of the fluid and the pressure is therefore continuous (cf. (2.6) and (3.4)); surface tension and the shape of the fluid front over the gap are not considered. A similar set of conditions was used by Aristoff et al in a related problem [1].

Whilst (3.4) is straightforward to solve analytically, the solution takes a particularly simple form if only one of the bending and tension terms are present. With only the bending term $(\mathcal{T}=0)$, the solution satisfying $h=h_{x}=h_{x x}=0$ at $x=L$ is

$$
h=\frac{\sigma}{24 \mathcal{B}}(L-x)^{3}(L-x-\ell)+h(X)(L-x)^{3} / \ell^{3},
$$



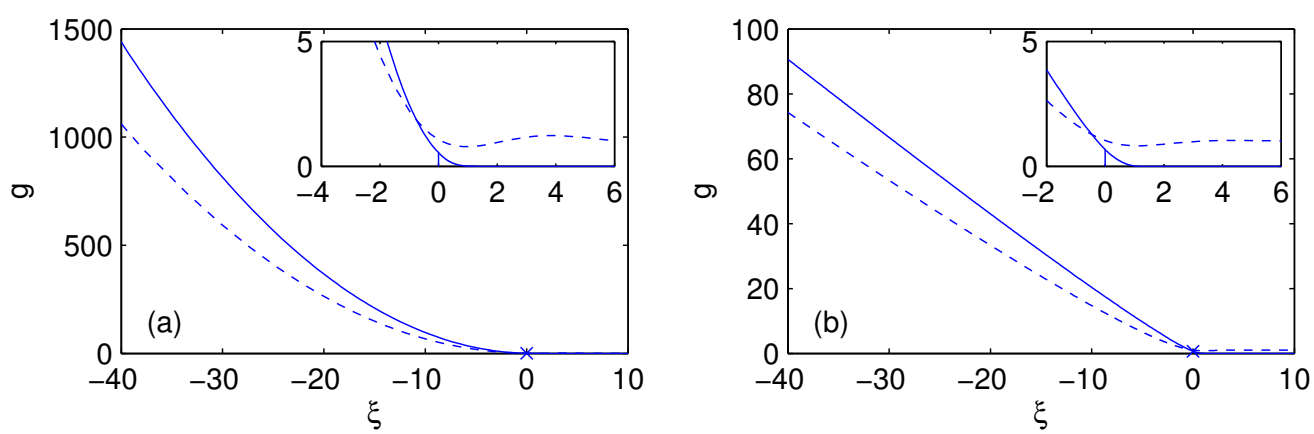

Figure 2. Boundary layer solutions for (a) bending and (b) tension. Dashed lines are for the pre-wetted film, solid lines are for the fluid lag, and the crosses indicate the position of the fluid edge. Insets show a magnification close to $\xi=0$.

where $\ell=L-X$ is the unknown 'lag'. The remaining continuity conditions are then

$$
\begin{array}{ll}
h_{x}(X)=\sigma \ell^{3} / 24 \mathcal{B}-3 h(X) / \ell, & h_{x x}(X)=-\sigma \ell^{2} / 4 \mathcal{B}+6 h(X) / \ell^{2}, \\
h_{x x x}(X)=3 \sigma \ell / 4 \mathcal{B}-6 h(X) / \ell^{3}, & h_{x x x x}(X)=-\sigma / \mathcal{B},
\end{array}
$$

which serve to determine $\ell$ and to provide three boundary conditions at $x=X$. Note that $h=O\left(\sigma \ell^{4}\right)$, so the lag distance becomes small for $h \rightarrow 0$ and $\sigma \gg 1$.

If only tension is included $(\mathcal{B}=0)$ the equation is lower order and we can demand only $h=h_{x}=0$ at $x=L$, and continuity of $h, h_{x}$ and $h_{x x}$ at $x=X$. The equation (3.4) then has solution

$$
h=\frac{\sigma}{2 \mathcal{T} N}(L-x)^{2},
$$

and the continuity conditions at $x=X$ are

$$
h(X)=\sigma \ell^{2} / 2 \mathcal{T} N, \quad h_{x}(X)=-\sigma \ell / \mathcal{T} N, \quad h_{x x}(X)=\sigma / \mathcal{T} N,
$$

which determine $\ell$ and provide two boundary conditions at $x=X$.

The numerical method with this regularization consists of first mapping the area occuppied by the expanding fluid onto a fixed domain, applying the boundary conditions (3.6) or (3.8) at each edge, and evolving those positions using (3.1). We then discretize the mapped domain, approximating derivatives with centred differences and evaluating $N$ with quadrature, and advance the system in time with the stiff integrator. A finite width and nonzero depth are required to initialize the computation. We therefore adopt the distributed source $w(x)$, taking $x_{v}=1$ and the initial $h$ to be a small bulge with a quartic shape spanning the vent. For currents on a horizontal surface, we again exploit symmetry and compute the solution over only half of the domain, $0 \leq x \leq X(t) \equiv X_{d}(t)$.

For the problems on a slope, the upslope and downslope regions, $-X_{u}(t) \leq x \leq 0$ and $0 \leq x \leq X_{d}(t)$, are mapped separately onto fixed grids.

\subsection{The boundary layers at the fluid fronts}

In the following sections we focus on the effect of the regularizations as the pre-wetted film becomes thin, $\delta \ll 1$, or as the pressure in the lag region becomes large and negative, $\sigma \gg 1$. In either limit, the regularization is felt primarily over a thin boundary layer 
adjoining the fluid fronts, $x=X(t)$. The short lengthscale of these boundary layers indicates that the term with highest derivative dominates the right-hand side of the balance in (3.2). We therefore rescale the variables according to

$$
x=X(t)+\varepsilon \xi, \quad h(x, t)=\Delta g(\xi),
$$

where $(\varepsilon, \Delta) \ll 1$. These small parameters can be chosen so that

$$
\dot{X} \varepsilon^{5}=\mathcal{B} \Delta^{3} \quad \text { if } \mathcal{B} \neq 0, \quad \text { or } \quad \dot{X} \varepsilon^{3}=\mathcal{T} N \Delta^{3} \text { if } \mathcal{B}=0,
$$

and either $\Delta=\delta$ for the pre-wetted film or $\Delta=\sigma \varepsilon^{4} / \mathcal{B}$ for the fluid lag (in view of the depth scale $h=O\left(\sigma \ell^{4} / \mathcal{B}\right)$ over the gas-filled gap; if $\mathcal{B}=0, \Delta=\sigma \varepsilon^{2} / \mathcal{T} N$ instead). The function $g(\xi)$ then satisfies

$$
-g_{\xi}=\left(g^{3} g_{\xi \xi \xi \xi \xi}\right)_{\xi} \quad(\mathcal{B} \neq 0) \quad \text { or } \quad g_{\xi}=\left(g^{3} g_{\xi \xi \xi}\right)_{\xi} \quad(\mathcal{B}=0) .
$$

The boundary conditions to be imposed on (3.11) depend on the choice of regularization. The details of the problems for both of our regularizations and the two choices, $\mathcal{B} \neq 0$ and $\mathcal{B}=0$, are relegated to Appendix A; figure 2 summarizes the four possible boundary layer solutions. The limiting behaviour as $\xi \rightarrow-\infty$ is either

$$
g \sim \frac{1}{2} \Gamma \xi^{2} \quad \text { or } \quad g \sim-3^{1 / 3} \xi(\ln -\xi)^{1 / 3},
$$

depending on whether bending or tension dominates the pressure gradient.

Rewriting this limiting behaviour in terms of the original variables, we derive the following matching conditions on the bulk of the flow at $x=X(t)$ : if bending is included,

$$
h=0, \quad h_{x}=0, \quad h_{x x}= \begin{cases}\Gamma \delta^{-1 / 5} \mathcal{B}^{-2 / 5} \dot{X}^{2 / 5} & \text { (pre-wetted film) }, \\ \bar{\Gamma} \sigma^{1 / 7} \mathcal{B}^{-3 / 7} \dot{X}^{2 / 7} & \text { (fluid lag) },\end{cases}
$$

where $\Gamma \approx 1.35$ and $\bar{\Gamma} \approx 1.77$ are numerically determined constants. If $\mathcal{B}=0$,

$$
h=0, \quad h_{x}= \begin{cases}-3^{1 / 3}(\ln 1 / \delta)^{1 / 3} \mathcal{T}^{-1 / 3} N^{-1 / 3} \dot{X}^{1 / 3} & \text { (pre-wetted film) }, \\ -3^{1 / 3}(\ln \sigma)^{1 / 3} \mathcal{T}^{-1 / 3} N^{-1 / 3} \dot{X}^{1 / 3} & \text { (fluid lag). }\end{cases}
$$

If $N$ is constant, these final conditions represent Tanner's law for the dynamic contact angle at a moving contact line [26]. The equivalent conditions on the curvature in (3.13) represent a modified version of this law for higher derivatives [9]. As noted in the appendix, the conditions in (3.14) have assumed that $\mathcal{T}, N$ and $\dot{X}$ are $O(1)$, and ignored logarithmic corrections in these variables. This asymptotic limit is not valid for large times.

\section{Flat solutions with bending and gravity}

We concentrate first on the flat case in which only bending and gravity contribute to the pressure: $\mathcal{B}=\mathcal{G}=1$ and $\mathcal{T}=\mathcal{S}=0$. The governing equation is then

$$
h_{t}=\left[h^{3}\left(h_{x x x x x}+h_{x}\right)\right]_{x}+w .
$$

A numerically calculated solution is shown in figure 3 for the case of the pre-wetted film,

with $w$ taken as a line source at $x=0$. Two distinct phases of the injection can be 

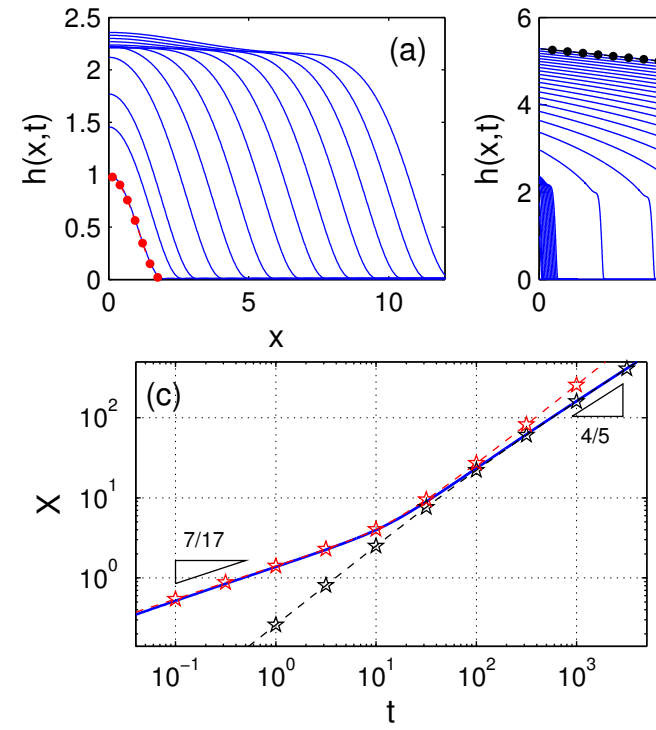
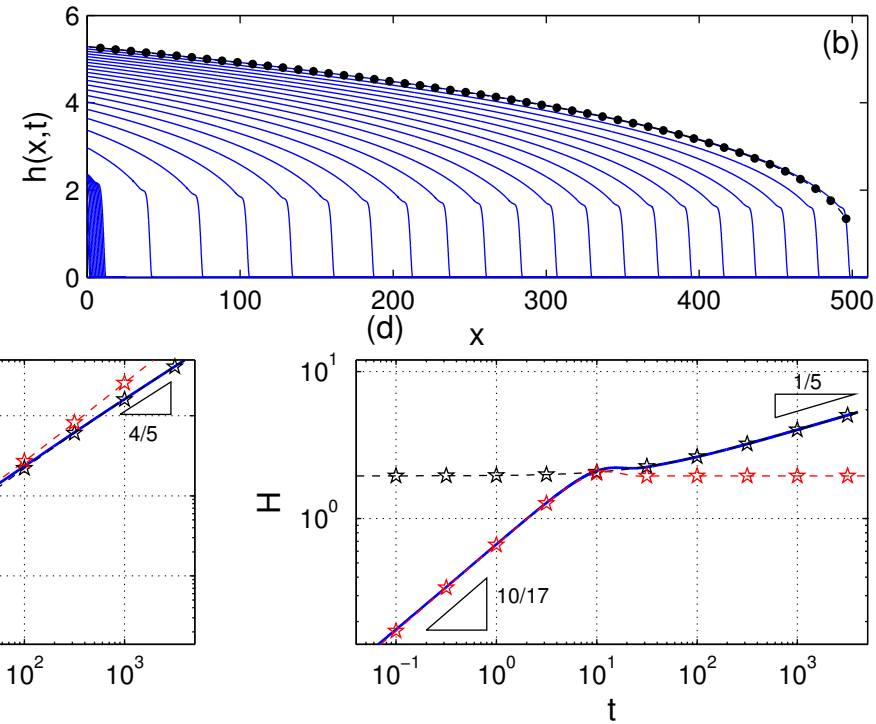

Figure 3. Numerical solutions for bending and gravity with the pre-wetted film with $\delta=10^{-2}$. Panel (a) shows snapshots of the solution at $t=2,4$ and $t=6,10, \ldots, 50$; panel (b) adds the snapshots for $t=200,400, \ldots, 4000$. Panel (c) shows the evolution of the fluid edge $x=X(t)$, and panel (d) shows the evolution of the central height $H=h(0, t)$. The lighter (red) pentagrams in panels (c) and (d) show the early time solution from section 4.1 (solution to (4.7)), and the dots in panel (a) show an example profile from that solution. Dark (black) pentagrams show the late time solution from section 4.2 (solution to (4.13), (4.14) and (4.18)), and the dots in panel (b) show the profile predicted at the final time from that solution.

identified: an 'early' time uniform pressure phase, in which spreading is controlled by the conditions at the edge, followed by a 'late' time evolution towards self-similar gravitydominated spreading. (We ignore the extremely early time behaviour, when the bulge height is comparable to the pre-wetted film thickness). Over each phase, characteristic temporal scalings emerge for the extent, $X(t)$, and central depth, $H(t)=h(0, t)$, of the current (figure $3(\mathrm{c})-(\mathrm{d})$ ).

\subsection{Early time $\delta^{9 / 5} \ll t \ll \delta^{-5 / 7}$ or $\sigma^{-9 / 11} \ll t \ll \sigma^{5 / 9}$ : uniform pressure}

During the early time evolution the fluid does not significantly spread laterally and the pressure is approximately uniform, $p \approx P(t)$, except very close to the edge [23]. The shape therefore evolves quasi-statically, with

$$
h_{x x x x}+h=P, \quad 0 \leq x \leq X(t) .
$$

The symmetry conditions $h_{x}=h_{x x x}=0$ apply at $x=0$, and the approximate boundary conditions from (3.13) at $x=X(t)$. The solution to (4.2) is

$$
h=\frac{H(C S+c s)}{(C-c)(S-s)}\left[1-\frac{(C s+S c)}{(C S+c s)} \cosh \frac{x}{\sqrt{2}} \cos \frac{x}{\sqrt{2}}-\frac{(C s+S c)}{(C S+c s)} \sinh \frac{x}{\sqrt{2}} \sin \frac{x}{\sqrt{2}}\right],
$$



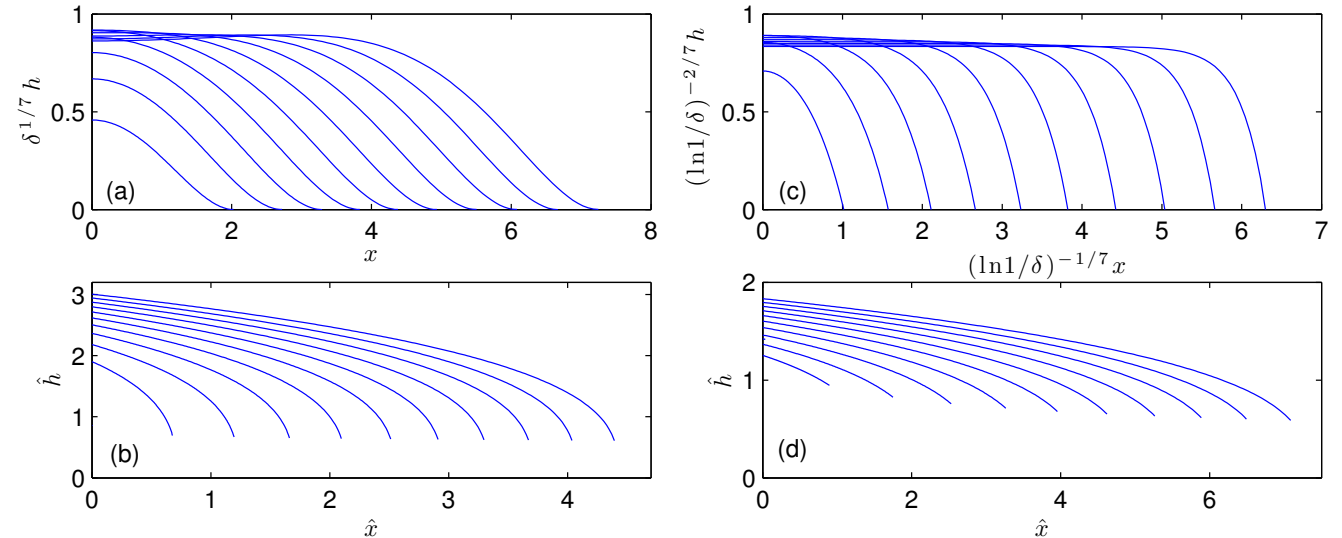

FIgURE 4. Asymptotic solutions for flat spreading. (a) Early time solution for bending and gravity, from (4.3)-(4.7) for $\delta^{1 / 7} t=1,2, \ldots, 10$. (b) Late time solution for bending and gravity, from (4.13), (4.14) and (4.18), for $\hat{t}=2,4, \ldots, 20$. (c) Early time solution for tension and gravity, from (5.3)-(5.6), for $(\ln 1 / \delta)^{3 / 7} t=1,2, \ldots, 10$. (d) Late time solution for tension and gravity, from (4.13), (4.14) and (5.16), for $\hat{t}=2,4, \ldots, 20$.

where $h(0, t) \equiv H(t)=P(t)(C-c)(S-s) /(C S+c s)$, and

$$
C=\cosh \frac{X}{\sqrt{2}}, \quad S=\sinh \frac{X}{\sqrt{2}}, \quad c=\cos \frac{X}{\sqrt{2}}, \quad s=\sin \frac{X}{\sqrt{2}} .
$$

The edge curvature condition from (3.13) is

$$
h_{x x}(X)=\frac{H(C S-c s)}{(C-c)(S-s)}= \begin{cases}\Gamma \delta^{-1 / 5} \dot{X}^{2 / 5} & \text { (pre-wetted film) } \\ \bar{\Gamma} \sigma^{1 / 7} \dot{X}^{2 / 7} & \text { (fluid lag) }\end{cases}
$$

Global mass conservation implies the volume constraint

$$
\frac{1}{2} t=\int_{0}^{X} h(x, t) \mathrm{d} x=H \frac{(C S+c s) X-\left(S^{2}+s^{2}\right) \sqrt{2}}{(C-c)(S-s)},
$$

in which we have used the solution (4.3) for $h$. Hence, on eliminating $H(t)$, the edge condition becomes

$$
\frac{(C S-c s) t}{4\left[(C S+c s) X-\left(S^{2}+s^{2}\right) \sqrt{2}\right]}= \begin{cases}\Gamma \delta^{-1 / 5} \dot{X}^{2 / 5} & \text { (pre-wetted film) } \\ \hat{\Gamma} \sigma^{1 / 7} \dot{X}^{2 / 7} & \text { (fluid lag) }\end{cases}
$$

which constitutes an ordinary differential equation for $X(t)$. The numerical solution to (4.7) is included in figure 3, and the evolving shape predicted by (4.3) is shown figure 4.

For small early times, $t \ll \delta^{-1 / 7}$ or $t \ll \sigma^{1 / 9}, X$ is relatively small and (4.3) reduces to the quartic

$$
h=H\left(1-x^{2} / X^{2}\right)^{2} .
$$

In this limit, (4.7) has the power-law behaviour

$$
X \sim\left\{\begin{array} { l l } 
{ A \delta ^ { 1 / 1 7 } t ^ { 7 / 1 7 } } & { } \\
{ \overline { A } \sigma ^ { - 1 / 2 3 } t ^ { 9 / 2 3 } }
\end{array} \quad H \sim \left\{\begin{array}{ll}
(15 / 16 A) \delta^{-1 / 17} t^{10 / 17} & \text { (pre-wetted film) } \\
(15 / 16 \bar{A}) \sigma^{1 / 23} t^{14 / 23} & \text { (fluid lag) },
\end{array}\right.\right.
$$


where

$$
A=(15 / 2 \Gamma)^{5 / 17}(17 / 7)^{2 / 17} \approx 1.84, \quad \bar{A}=(15 / 2 \bar{\Gamma})^{7 / 23}(23 / 9)^{2 / 23} \approx 1.68 .
$$

For larger early times, $\delta^{-1 / 7} \ll t \ll \delta^{-5 / 7}$ or $\sigma^{1 / 9} \ll t \ll \sigma^{5 / 9}$, the profile in (4.3) becomes almost uniform with $h \approx H$, and the solution tends towards

$$
X \sim\left\{\begin{array} { l l } 
{ \delta ^ { 1 / 7 } ( 4 \Gamma ) ^ { - 5 / 7 } t , } \\
{ \sigma ^ { - 1 / 9 } ( 4 \overline { \Gamma } ) ^ { - 7 / 9 } t , }
\end{array} \quad H \sim \left\{\begin{array}{ll}
\frac{1}{2} \delta^{-1 / 7}(4 \Gamma)^{5 / 7} & \text { (pre-wetted film) } \\
\frac{1}{2} \sigma^{1 / 9}(4 \bar{\Gamma})^{7 / 9} & \text { (fluid lag) }
\end{array}\right.\right.
$$

i.e. $H$ becomes constant and $X$ grows linearly in time, as seen in figure 3 .

Substituting these predictions into the original equation, one finds that constant pressure is established for $t \gg \delta^{9 / 5}$ or $t \gg \sigma^{-9 / 11}$, equivalent to the time taken for the current to become sufficiently long that the boundary layer develops at the contact line. Moreover, the approximation breaks down when $t=O\left(\delta^{-5 / 7}\right)$ or $t=O\left(\sigma^{7 / 9}\right)$, at which point the time derivative $h_{t}$ can no longer be neglected and a pressure gradient develops. At such times, the length of the current is relatively large, $X=O\left(\delta^{-4 / 7}\right)$ or $X=O\left(\sigma^{2 / 3}\right)$, indicating that bending becomes unimportant over the bulk of the fluid layer.

\subsection{Late time $t \gg \delta^{-1 / 7}$ or $t \gg \sigma^{1 / 9}$ : transition to gravity control}

For the late time behaviour, and concentrating for the moment on the pre-wetted film, we rescale the variables according to

$$
t=\delta^{-5 / 7} \hat{t}, \quad x=\delta^{-4 / 7} \hat{x}, \quad h=\delta^{-1 / 7} \hat{h}, \quad X=\delta^{-4 / 7} \hat{X} .
$$

To leading order, we then recover a standard equation describing viscous spreading under gravity:

with

$$
\hat{h}_{\hat{t}}=\left(\hat{h}^{3} \hat{h}_{\hat{x}}\right)_{\hat{x}}, \quad 0<\hat{x} \leq \hat{X}(\hat{t})
$$

$$
-\hat{h}^{3} \hat{h}_{\hat{x}}=\frac{1}{2} \quad \text { at } \quad \hat{x}=0, \quad \hat{X}_{\hat{t}}=-\hat{h}^{2} \hat{h}_{\hat{x}} \quad \text { at } \quad \hat{x}=\hat{X} .
$$

Unlike the problem considered by Huppert [15] and others, however, the fluid depth at the edge, $\hat{H}_{1} \equiv \hat{h}(\hat{X})$, is not zero. Instead, the bending term reasserts its influence over a layer adjacent to the front region; this edge layer connects the interior of the gravity current to the contact line and has a thickness of $\delta^{4 / 7}$ in terms of the new coordinate $\hat{x}$ (i.e. it is $O(1)$ in terms of $x$ ). Writing $\hat{x}=\hat{X}-\delta^{4 / 7} y$, the leading order equation over the edge layer is

$$
\hat{h}_{\text {yyyyy }}+\hat{h}_{y}=0 \quad \text { with } \quad \hat{h} \rightarrow \hat{H}_{1} \quad \text { as } \quad y \rightarrow \infty .
$$

(Matching to the slope of the interior solution occurs at higher order in the expansion of this edge layer solution, the details of which we omit for brevity). The conditions (3.13) require

$$
\hat{h}=\hat{h}_{y}=0, \quad \hat{h}_{y y}=\Gamma \hat{X}_{\hat{t}}^{2 / 5}, \quad \text { at } \quad y=0 .
$$

The solution is

$$
\hat{h}=\hat{H}_{1}\left[1-e^{-y / \sqrt{2}}\left(\cos \frac{y}{\sqrt{2}}+\sin \frac{y}{\sqrt{2}}\right)\right],
$$


with

$$
\hat{H}_{1}=\Gamma \hat{X}_{\hat{t}}^{2 / 5}
$$

This last result determines the extra condition required to solve the interior problem in (4.13)-(4.14). A numerical solution is required (but is more straightforward than for the original sixth-order equation) and is shown in figure 4 . The evolution of the fluid edge and centre height from this solution are also included in figure 3. The initial condition for the calculation (begun at small, but finite $\hat{t}$ ) is taken from the limiting behaviour of the early time solution in section 4.1: $\hat{h}(\hat{x}, 0)=\frac{1}{2}(4 \Gamma)^{5 / 7}$ is uniform while $\hat{X} \sim(4 \Gamma)^{-5 / 7} \hat{t}$.

As $\hat{t} \rightarrow \infty$, the front decelerates and the edge height $\hat{H}_{1}$ tends towards zero. The solution then converges towards the similarity solution of (4.13)-(4.14) that results when $\hat{h}=0$ at $\hat{x}=\hat{X}$. In terms of the original variables, that similarity solution,

$$
h \rightarrow t^{1 / 5} f\left(x / t^{4 / 5}\right)
$$

satisfies

$$
\frac{1}{5} f-\frac{4}{5} \eta f_{\eta}=\left(f^{3} f_{\eta}\right)_{\eta}, \quad-f^{3} f_{\eta}=\frac{1}{2} \quad \text { at } \quad \eta=0, \quad-f^{3} f_{\eta}=f=0 \quad \text { at } \quad \eta=\eta_{1} .
$$

The bending term causes a region of adjustment from this solution close to the edge, which is eventually subsumed into the pre-wetted film. The very late time spreading $\left(t \gg \delta^{-5 / 7}\right)$ is therefore given by

$$
X \sim \eta_{1} t^{4 / 5}, \quad H \sim f(0) t^{1 / 5},
$$

where $\eta_{1} \approx 0.66$ and $f(0) \approx 1.00$ are determined by numerical solution of (4.20).

For the case of the fluid lag, the relevant scalings are

$$
t=\sigma^{5 / 9} \hat{t}, \quad x=\sigma^{4 / 9} \hat{x}, \quad h=\sigma^{1 / 9} \hat{h}, \quad X=\sigma^{4 / 9} \hat{X}, \quad w=\delta^{-4 / 9} \hat{w},
$$

and the leading-order problem to solve is then the same as (4.13)-(4.14), but with the boundary condition (4.18) replaced by

$$
\hat{H}_{1}=\bar{\Gamma} \hat{X}_{\hat{t}}^{2 / 7} .
$$

\subsection{Pure bending}

If only the bending term controls the spreading and there is no gravity, the quartic shape from (4.8) and the power-law behaviour in (4.9) apply indefinitely. There are two notable features of this solution.

First, spreading is entirely controlled by the behaviour close to the contact line. The smaller the pre-wetted film or the more negative the lag pressure, the more the lateral spreading of the fluid is confined and the greater the height at the centre. There is no convergence as $\delta \rightarrow 0$ or $\sigma \rightarrow \infty$; the prediction in that limit is an infinitely narrow, infinitely tall blister. The dependence on either $\delta$ or $\sigma$ is, however, rather weak $\left(\delta^{1 / 17}\right.$ or $\sigma^{-1 / 23}$ ), and for practical purposes the difference is relatively small for different values of the regularization parameters; see figures 5 and 6 . These figures show numerical solutions to the full problem with only the bending term in the equation, for three different values of the regularization parameters $\delta$ and $\sigma$, comparing the results with the predictions from (4.9). 

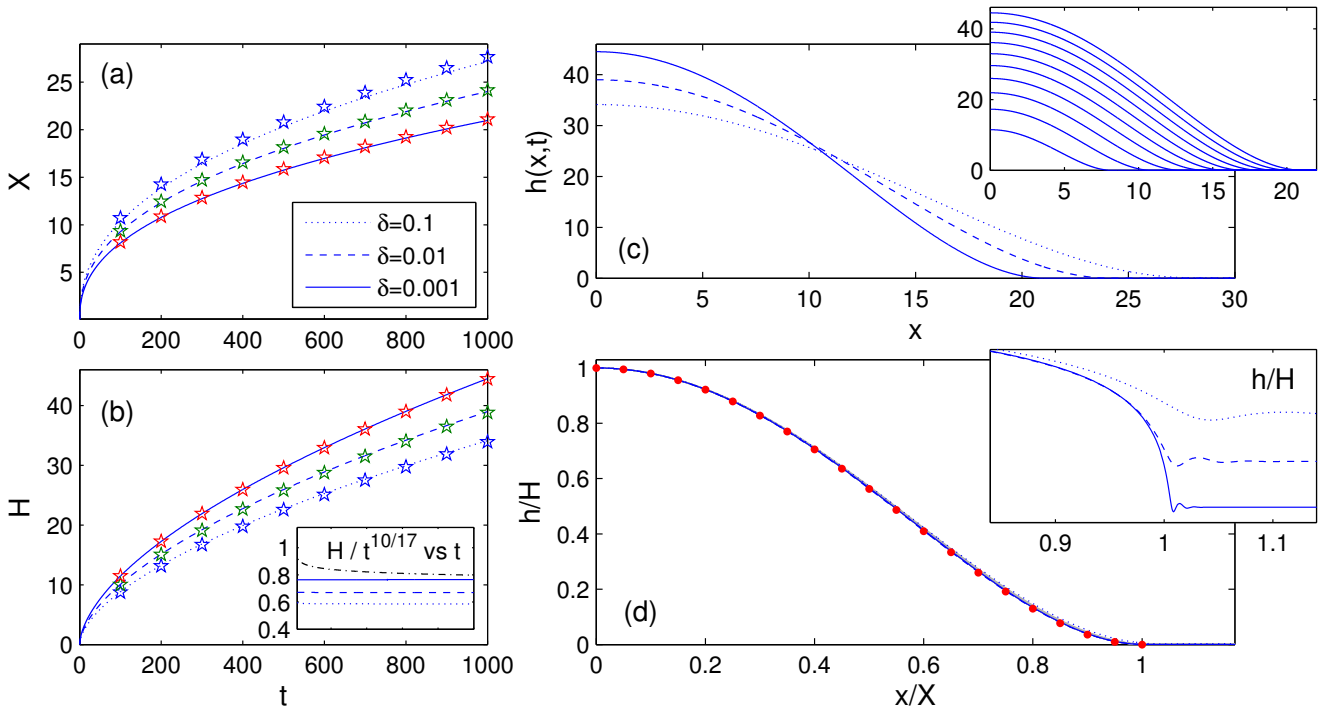

Figure 5. Numerical solutions for pure bending with the pre-wetted film. Panels (a) and (b) show the positions of the fluid edge $X(t)$ and the maximum deflection $H(t)$ for the three values of film thickness shown. Pentagrams show the predictions of the analysis in (4.9). The inset in (b) shows a compensated plot of $H / t^{10 / 17}$, with the black dash-dotted line showing the equivalent behaviour if $H \sim t^{5 / 9}$. Panel (c) shows final shapshots for the same solutions (main panel), and 10 snapshots of $h$ for the computation with $\delta=10^{-3}$. Panel (d) shows the same data as (c) (including the inset), plotted using the scaled variables (main panel), and a close up of the edge for the final snapshots (inset). The dots show $\left(1-x^{2} / X^{2}\right)^{2}$.
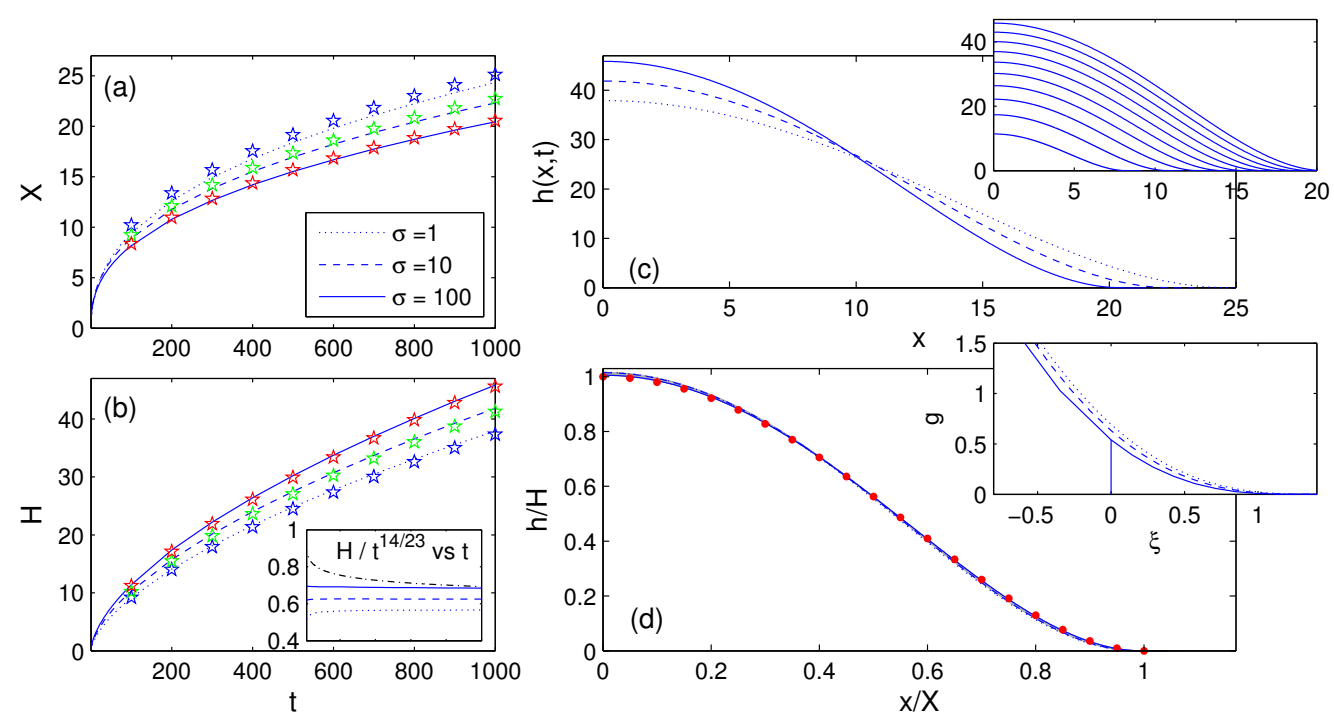

FIGURE 6. Numerical solutions for pure bending with the fluid lag, showing the same information as in figure 5, with the pentagrams showing the analytical result from (4.9). The inset in (b) shows a compensated plot of $H / t^{14 / 23}$, with the black dash-dotted line showing the equivalent behaviour if $H \sim t^{5 / 9}$. The inset in panel (d) shows a close up near the edge, plotted using the scaled coordinates of the boundary layer from appendix A. 
Second, the powers of time are different for the two regularizations, and in both cases are different from the $(X, H) \sim\left(t^{4 / 9}, t^{5 / 9}\right)$ scalings expected based purely on the scaleinvariance of the bending-dominated differential equation. That is, one does not observe a similarity solution. Nevertheless, the difference between these exponents is rather small. For example, for $X(t), 4 / 9 \approx 0.44,7 / 17 \approx 0.41$ and $9 / 23 \approx 0.39$. This coincidence is presumably responsible for the erroneous identification of a similarity solution in previous work [23]. The fact that the solutions do not tend towards the self-similar scaling suggests that a similarity solution to the bending problem does not in fact exist.

\section{Flat solutions with tension and gravity}

We now consider the case with only tension and gravity: $\mathcal{T}=\mathcal{G}=1$ and $\mathcal{B}=\mathcal{S}=0$. The equations to be solved are

$$
h_{t}=\left[h^{3}\left(-N h_{x x x}+h_{x}\right)\right]_{x}+w, \quad N=\frac{1}{L} \int_{0}^{L} \frac{1}{2} h_{x}^{2} \mathrm{~d} x .
$$

A numerical solution is shown in figure 7 for the case of the pre-wetted film. As for the bending solution in figure 3, the injection can be broken down into an early uniform pressure phase, controlled by the conditions at the fluid edge, and a subsequent transition towards self-similar gravity-controlled spreading, with tension and the contact line playing no role.

As suggested by the matching conditions in section 3.4, the asymptotic analysis of the solutions in this case relies upon expansions in powers of $\ln 1 / \delta$ or $\ln \sigma$. For practical purposes, these numbers are not very large and the approximations cannot be expected to be as good as for the bending case in the previous section. Nevertheless, the analysis serves a useful purpose to understand the role of the regularization in controlling the spreading.

\subsection{Early time $t \ll(\ln 1 / \delta)^{15 / 14}$ or $t \ll(\ln \sigma)^{15 / 14}$ : uniform pressure}

The approximate solution for the early pressure phase is constructed in much the same way as for the bending problem in section 4.1. For a constant pressure $p \approx P(t)$ over the interior, we have

$$
-N h_{x x}+h=P, \quad 0 \leq x \leq X(t),
$$

with the symmetry condition $h_{x}=0$ at $x=0$, and the conditions (3.14) at $x=X$. These are combined with the global volume constraint and the integral expression for the tension $N(t)$ in (5.1), to provide an evolution equation for $X(t)$.

The solution of (5.2) is

$$
h=H \frac{\cosh (X / \sqrt{N})-\cosh (x / \sqrt{N})}{\cosh (X / \sqrt{N})-1},
$$

where $H(t)=P(t)[\cosh (X / \sqrt{N})-1] / \cosh (X / \sqrt{N})$. The tension from (5.1) is therefore related to $H$ and $X$ by the transcendental equation

$$
N=\frac{H^{2}}{4 X \sqrt{N}} \frac{\sinh (X / \sqrt{N}) \cosh (X / \sqrt{N})-X / \sqrt{N}}{[\cosh (X / \sqrt{N})-1]^{2}} .
$$



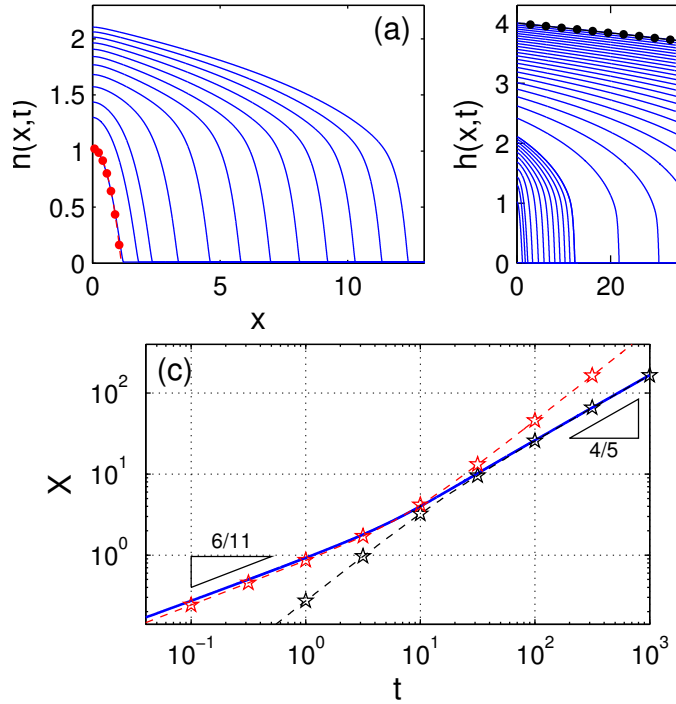
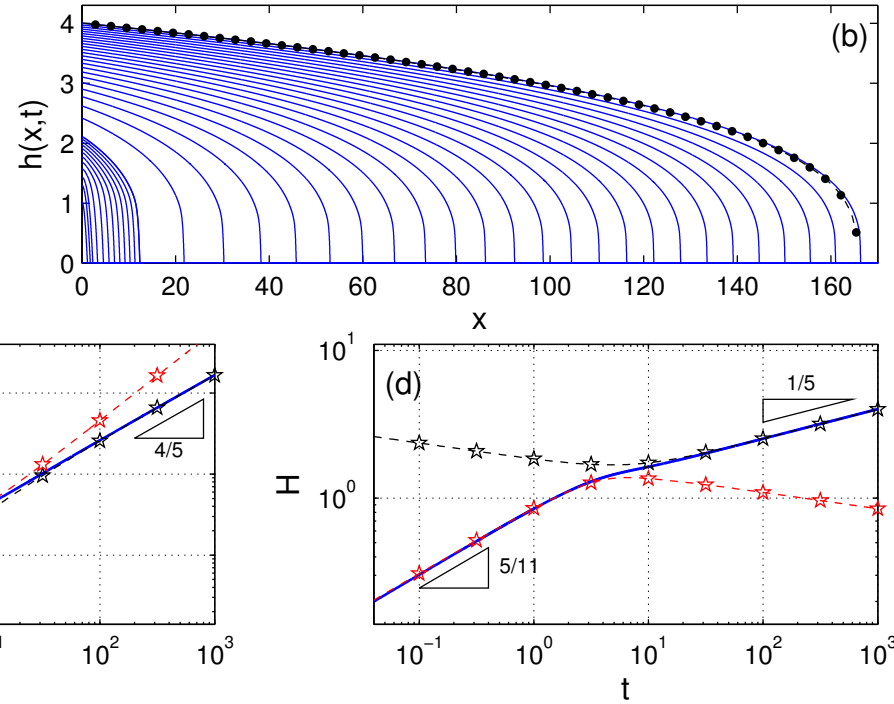

FiguRE 7. Numerical solutions for tension and gravity with the pre-wetted film with $\delta=10^{-2}$. Panel (a) shows snapshots of the solution at $t=1.6,3.2,4.8$ and $t=8,12, \ldots, 40$; panel (b) adds snapshots for $t=80,120, \ldots, 1000$. Panel (c) shows the evolution of the fluid edge $x=X$, and panel (c) shows the evolution of the central height $H=h(0, t)$. Lighter (red) pentagrams in panels (c) and (d) show the approximate early time solution from section 5.1, and the dots in panel (a) show a sample profile from that solution. Dark (black) pentagrams show the approximate late time solution from section 5.2, and the dots in panel (b) show the profile predicted at the final time from that solution.

The global volume constraint demands

$$
\frac{1}{2} t=\int_{0}^{X} h(x, t) \mathrm{d} x=H \sqrt{N} \frac{(X / \sqrt{N}) \cosh (X / \sqrt{N})-\sinh (X / \sqrt{N})}{\cosh (X / \sqrt{N})-1},
$$

and matching to the slope at the contact line in (3.14) requires

$$
\frac{H \sinh (X / \sqrt{N})}{\sqrt{N}[\cosh (X / \sqrt{N})-1]}=3^{1 / 3} \dot{X}^{1 / 3} N^{-1 / 3} \times\left\{\begin{array}{l}
(\ln 1 / \delta)^{1 / 3} \\
(\ln \sigma)^{1 / 3}
\end{array}\right.
$$

Combining (5.4), (5.5) and (5.6) leads to an evolution equation for $X(t)$, the solution to which is shown in figures 4 and 7 . Given that we may simply replace $\delta$ with $\sigma^{-1}$ to recover the fluid lag problem from the prewetted film case, we continue with only the latter.

For small early times, $t \ll(\ln 1 / \delta)^{3 / 7}, X$ is small and the shape in (5.3) is approximately

$$
h=H\left(1-x^{2} / X^{2}\right) .
$$

In this limit the solutions for $X$ and $H$ are

$$
X \sim C(\ln 1 / \delta)^{-1 / 11} t^{6 / 11}, \quad H \sim(3 / 4 C)(\ln 1 / \delta)^{1 / 11} t^{5 / 11},
$$

where

$$
C=(11 / 6)^{1 / 11}(3 / 4)^{3 / 11} \approx 0.98 .
$$


For larger early times, $(\ln 1 / \delta)^{3 / 7} \ll t \ll(\ln 1 / \delta)^{15 / 14}$, the counter-intuitive limiting behaviour is

$$
X \sim(3 / 10)^{1 / 3}(1 / 2)^{5 / 9}(\ln 1 / \delta)^{-1 / 3} t^{10 / 9}, \quad H \sim(10 / 3)^{1 / 3}(1 / 2)^{4 / 9}(\ln 1 / \delta)^{1 / 3} t^{-1 / 9} ;
$$

i.e. $H$ is decreasing and $X$ is growing faster than linearly (see figure 7 ). This reflects a slumping behaviour, when the weight of the bulge becomes sufficient to overcome the tension holding it together, and more fluid spreads outwards from the centre than is provided from the source alone. However, this aspect of the solution lasts only a brief time before the constant-pressure approximation itself breaks down; it is not seen in the full numerical solutions with our modest values of $\ln 1 / \delta$.

\subsection{Late time $t \gg(\ln 1 / \delta)^{3 / 7}$ or $t \gg(\ln \sigma)^{3 / 7}$ : transition to gravity control}

As in the bending problem, at later times the interior of the flow becomes dominated by gravity alone and the tension term is important only in a narrow layer close to the fluid edge. We write

$$
\begin{gathered}
t=(\ln 1 / \delta)^{15 / 14} \hat{t}, \quad x=(\ln 1 / \delta)^{6 / 7} \hat{x}, \quad h=(\ln 1 / \delta)^{3 / 14} \hat{h}, \\
X=(\ln 1 / \delta)^{6 / 7} \hat{X}, \quad N=(\ln 1 / \delta)^{-2 / 7} \hat{N} .
\end{gathered}
$$

The interior problem is then identical to that given in (4.13)-(4.14), except for the edge condition $H_{1}$. The depth at the edge, $\hat{H}_{1}=\hat{h}(\hat{X})$ is determined by matching to the edge layer, in which we write $\hat{x}=\hat{X}-(\ln 1 / \delta)^{-1} \hat{y}$ and solve the leading order equation,

$$
-\hat{N} \hat{h}_{\hat{y} \hat{y} \hat{y}}+\hat{h}_{\hat{y}}=0 \quad \text { with } \quad \hat{h} \rightarrow \hat{H}_{1} \quad \text { as } \quad \hat{y} \rightarrow \infty,
$$

with the conditions (3.14) requiring

$$
\hat{h}=0, \quad \hat{h}_{\hat{y}}=3^{1 / 3} \hat{X}_{\hat{t}}^{1 / 3} \hat{N}^{-1 / 3} \quad \text { at } \quad \hat{y}=0 .
$$

The solution is

$$
\hat{h}=\hat{H}_{1}\left(1-e^{-\hat{y} / \sqrt{\hat{N}}}\right), \quad \hat{H}_{1}=3^{1 / 3} \hat{X}_{\hat{t}}^{1 / 3} \hat{N}^{1 / 6} .
$$

The tension $\hat{N}$ is dominated by the stretching contribution from the edge layer and is given by

$$
\hat{N}=\frac{1}{\hat{X}} \int_{0}^{\infty} \frac{1}{2} \hat{h}_{\hat{y}}^{2} \mathrm{~d} \hat{y} \quad \rightarrow \quad \hat{N}=\frac{\hat{H}_{1}^{4 / 3}}{4^{2 / 3} \hat{X}^{2 / 3}} .
$$

The expression for the fluid depth at the border of the interior region is therefore

$$
\hat{H}_{1}=\frac{3^{3 / 7}}{4^{1 / 7}} \hat{X}^{-1 / 7} \hat{X}_{\hat{t}}^{3 / 7} .
$$

The interior problem (4.13)-(4.14) with (5.16) once again requires a numerical solution; initial conditions (imposed at small, but finite $\hat{t}$ ) follow from the limiting behaviour in section 5.1: $\hat{X}(\hat{t}) \sim(3 / 10)^{1 / 3}(1 / 2)^{5 / 9} \hat{t}^{10 / 9}$ and $\hat{h}(\hat{x}, \hat{t}) \sim(10 / 3)^{1 / 3}(1 / 2)^{4 / 9} \hat{t}^{-1 / 9}$. The solution is shown in figures 4 and 7 . Eventually, for $\hat{t} \rightarrow \infty$, the edge depth $\hat{H}_{1}$ tends towards zero and the behaviour converges to the similarity solution in (4.20) with the long-time spreading given by (4.21). 

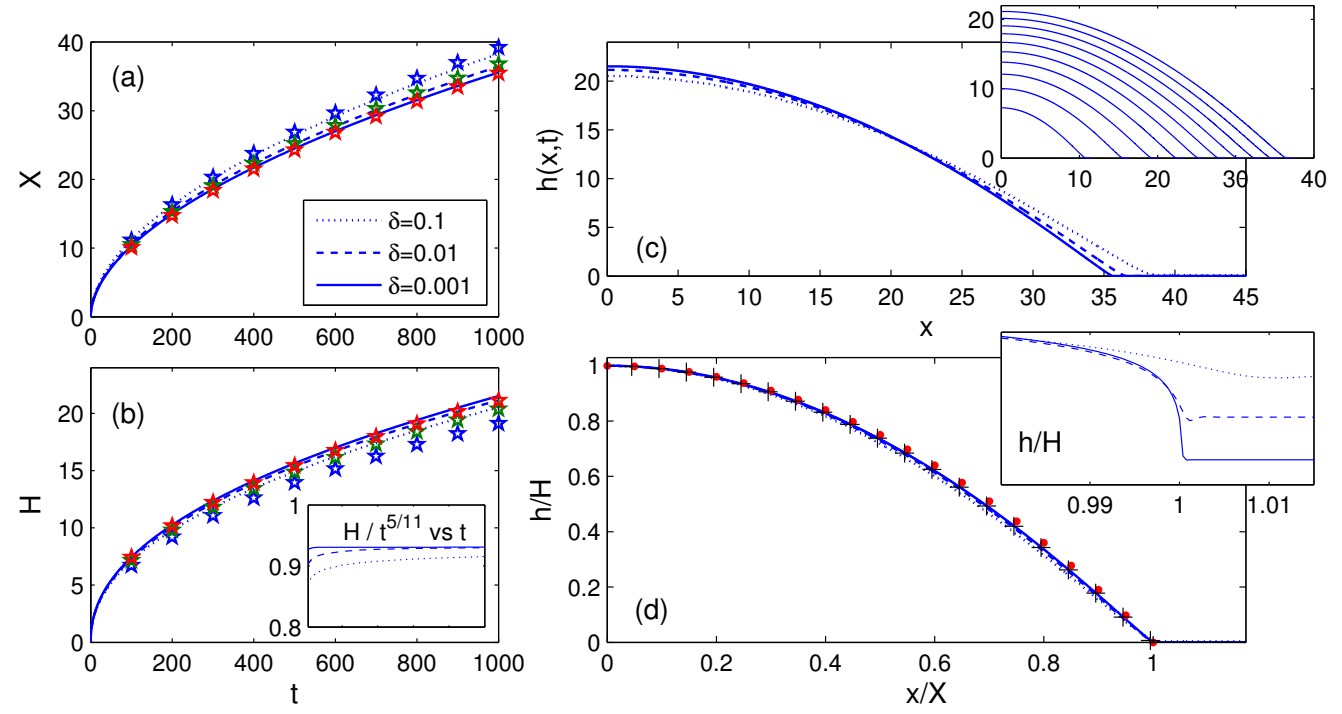

Figure 8. Numerical solutions for pure tension with a pre-wetted film. Panels (a) and (b) show $X(t)$ and $H(t)$; the pentagrams plot the predictions from (5.8) for the three values of $\delta$. The inset in panel (b) shows a compensated plot of $H / t^{5 / 11}$. Panel (c) shows the final profile for each case; the inset shows ten earlier snapshots for the computation with $\delta=10^{-3}$. Panel (d) shows the data from (c) in the scaled variables, $h / H$ and $x / X$, with the inset displaying a magnification close to the final fluid fronts. Dots show $\left(1-x^{2} / X^{2}\right)$ and crosses show the similarity solution from (5.17)-(5.18) for $\delta=10^{-1}$.

\subsection{Pure tension}

If there is no gravity term, the quadratic solution (5.7) for the constant pressure phase and the power laws in (5.8) apply indefinitely. As for the pure bending problem, this implies that the spreading is controlled by the pre-wetted film depth or lag pressure, but the dependence on $\delta$ or $\sigma$ is even weaker than before. Some numerical solutions are shown in figure 8 for different values of the regularization parameters; there is almost no difference between the solutions and in fact the difference is less than predicted by the result in (5.8) (this is particularly clear in the plots of $H$ ). This is presumably because that analysis relies upon the largeness of $\ln 1 / \delta$ or $\ln \sigma$, and even for $\delta=10^{-3}$ this approximation is rather poor. The disagreement may also be linked to the neglect of the time-dependent terms in the logarithm of the matching behaviour in (3.14) (see Appendix A.3); the numerical solutions continue for long enough that such terms are comparable to $1 / \delta$ or $\sigma$ and should be taken into account. Nevertheless, even without accommodating this long time behaviour, the analysis indicates that there is no convergence to a limit for $\delta \rightarrow 0$ or $\sigma \rightarrow \infty$.

Note that the power laws predicted by $(5.8),(X, H) \sim\left(t^{6 / 11}, t^{5 / 11}\right)$ are the same as those predicted by a similarity scaling of the equation with pure tension (which are different from those predicted for surface tension [18] because $N \sim t^{-2 / 11}$ ). This reflects the fact that, for the approximate solution in (5.8), the time derivative $h_{t}$ is $O(\ln 1 / \delta)^{-1}$ smaller than the divergence of the flux, regardless of time. An alternative analysis of the pure tension case is to treat $\ln 1 / \delta$ as $O(1)$, thereby invalidating the uniform pressure 

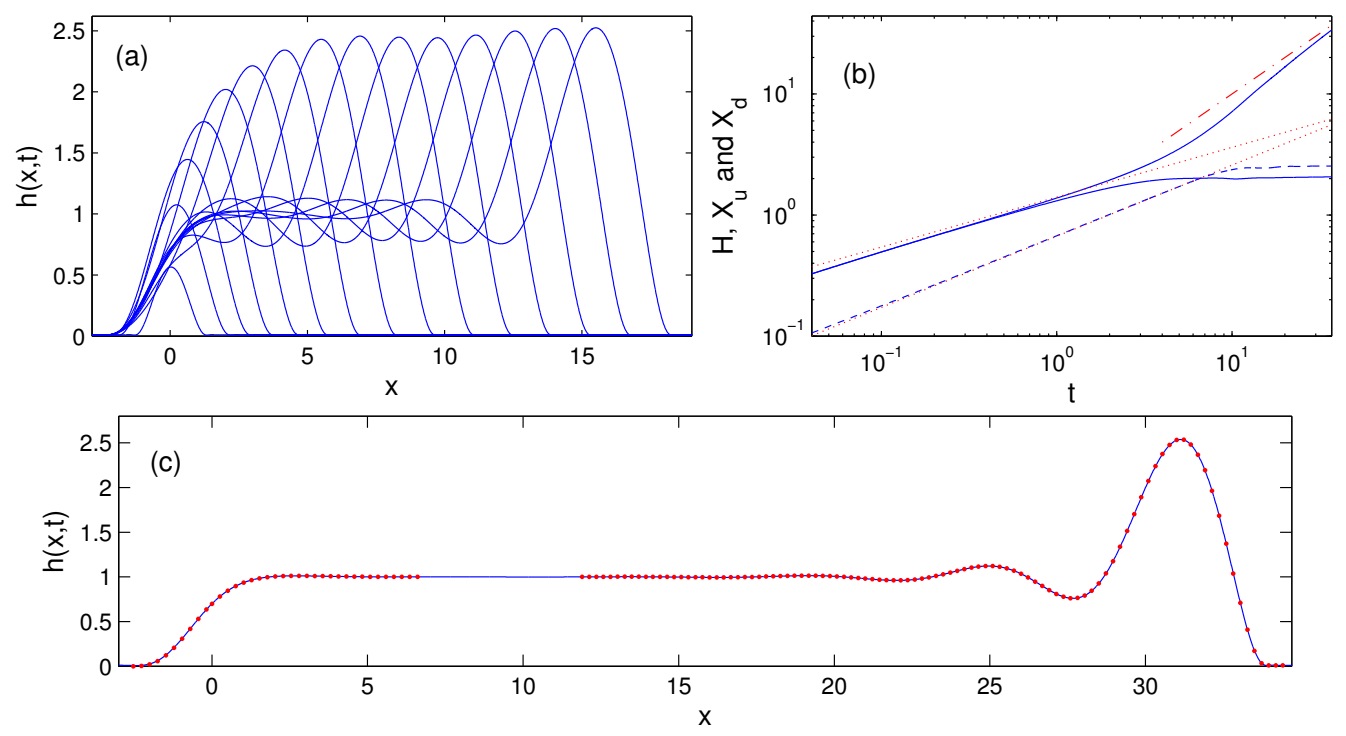

Figure 9. Numerical solutions for bending, slope and a pre-wetted film with $\delta=10^{-2}$. Panel (a) shows snapshots at $t=1.5,3,4.5, \ldots, 21$. Panel (b) shows the evolution of the downstream and upstream fluid edges, $X_{d}(t)$ and $X_{u}(t)$ (solid), and maximum height $H$ (dashed). The dotted lines show the early time behaviour from (5.8); the dot-dashed line shows the eventual linear trend of $X_{d}(t)$. Panel (c) shows the final profile, with dots showing the final upstream shape from (6.2) and the travelling wave shape from (6.6). The travelling-wave solution is positioned so that its maximum lines up with that of the numerical solution.

approximation. The effective boundary conditions in (3.14) remain valid (the boundary layer analysis in Appendix A relies on $\delta$ being small, rather than $\left.(\ln 1 / \delta)^{-1}\right)$, and the problem then has a similarity solution $h=t^{5 / 11} f\left(x / t^{6 / 11}\right), N=t^{-2 / 11} \nu$, where $f(\eta), \nu$, and the scaled fluid edge position $\eta_{1}$, satisfy

$$
\frac{5}{11} f-\frac{6}{11} \eta f_{\eta}=\nu\left[f^{3} f_{\eta \eta \eta}\right]_{\eta}, \quad \nu=\frac{1}{\eta_{1}} \int_{0}^{\eta_{1}} \frac{1}{2} f_{\eta}^{2} \mathrm{~d} \eta
$$

with

$$
\begin{gathered}
f_{\eta}=0, \quad \nu f^{3} f_{\eta \eta \eta}=\frac{1}{2} \quad \text { at } \eta=0 \\
f^{3} f_{\eta \eta \eta}=0, \quad f=0, \quad f_{\eta}=-(18 / 11)^{1 / 3}(\ln 1 / \delta)^{1 / 3} \eta_{1}^{1 / 3} \nu^{-1 / 3} \quad \text { at } \quad \eta=\eta_{1} .
\end{gathered}
$$

Note that this similarity solution still depends on the film depth $\delta$. A numerical solution to $(5.17)-(5.19)$ is included in figure 6 and is very close to the quadratic in (5.7).

\section{Sloping solutions with bending}

We now consider injection on an incline with bending stresses. To keep the discussion concise we ignore all other contributions to the pressure and set $\mathcal{B}=\mathcal{S}=1$ and $\mathcal{T}=\mathcal{G}=$ 0. Hence,

$$
h_{t}=\left[h^{3}\left(h_{x x x x x}-1\right)\right]_{x}+w .
$$


A numerical solution with the pre-wetted film is shown in figure 9. Initially, the behaviour is the same as for the flat solution of section 4, the slope having little effect until the length of the current becomes sufficiently large. At longer length scales, the slope dominates the pressure gradient over most of the flow, so that $h \approx 1$ there (the constant injection providing a downslope flux of unity). The bending stress remains important close to the vent, where it controls the distance that the fluid spreads upslope and its eventual limit $X_{u}(\infty)$, and close to the downslope fluid front $x=X_{d}(t)$, where it controls the shape of a travelling wave and gives rise to a decaying oscillation leading back from the front.

\subsection{Upstream spreading}

After the initial transient, the shape at the upstream end tends to a steady state. Ignoring the pre-wetted film, and for a line source at $x=0$, the steady profile is given by

$$
h_{x x x x x}= \begin{cases}1 & -X_{u}<x<0, \\ 1-1 / h^{3} & 0<x,\end{cases}
$$

subject to

$$
h=h_{x}=h_{x x}=0 \quad \text { at } \quad x=-X_{u}(\infty),
$$

continuity of $h$ and its first four derivatives at $x=0$, and $h \rightarrow 1$ as $x \rightarrow \infty$. The numerical solution to this problem indicates $X_{u}(\infty) \approx 2.53$, and is included in figure $9(\mathrm{c})$.

\subsection{The travelling wave}

We examine the behaviour at the downstream edge by moving to a translating frame with the coordinate

$$
\zeta=x-X_{d}(t) .
$$

In this frame, the solution evolves to a steady travelling wave determined by

$$
-\dot{X}_{d} h^{\prime}=\left[h^{3}\left(h^{\prime \prime \prime \prime \prime}-1\right)\right]^{\prime},
$$

where the prime denotes a derivative with respect to $\zeta$.

In the case of the prewetted film, it is necessary to account for the small flux of fluid in the film ahead of the front (neglected above in (6.2)), which demands that the front speed is $\dot{X}_{d}=1+\delta+\delta^{2}$. Equation (6.5) can then be integrated to give

$$
h^{\prime \prime \prime \prime \prime \prime}=1-\frac{1+\delta+\delta^{2}}{h^{2}}+\frac{\delta+\delta^{2}}{h^{3}},
$$

subject to

$$
h \rightarrow 1 \quad \text { as } \quad \zeta \rightarrow-\infty \text { and } h \rightarrow \delta \quad \text { as } \quad \zeta \rightarrow \infty .
$$

A solution to this steady problem is included in figure 9 .

In the case of the fluid lag, for which the fluid loses contact with the elastic sheet at $\zeta=0$, the front speed is $\dot{X}_{d}=1$ and the integral of (6.5) becomes

$$
h^{\prime \prime \prime \prime \prime}=1-\frac{1}{h^{2}},
$$



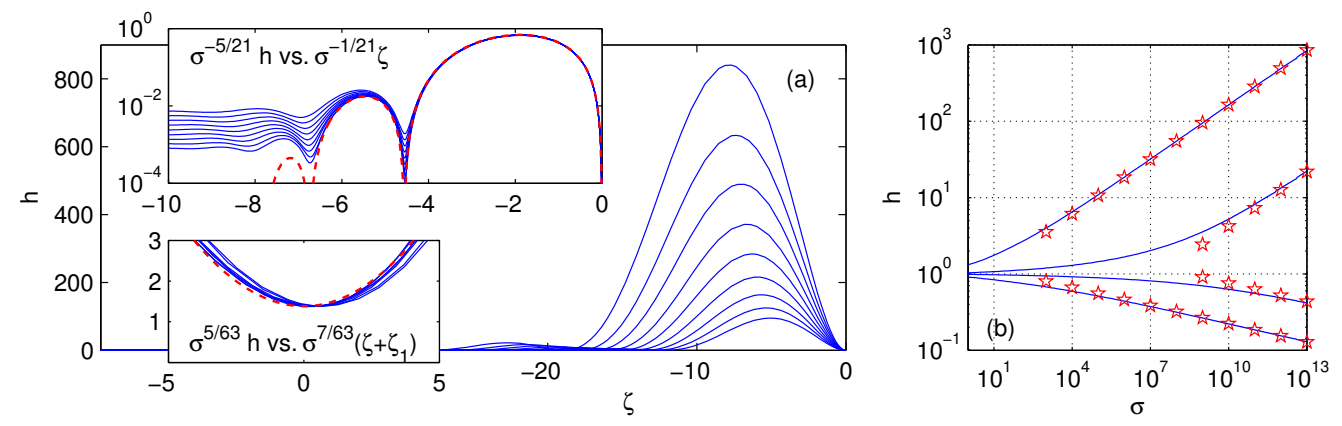

FiguRE 10. Numerically computed travelling waves with the fluid lag. Panel (a) shows front profiles for $\sigma=10^{9}, 10^{9.5}, \ldots, 10^{13}$; the upper inset shows the same data logarithmically, with $x$ scaled by $\sigma^{1 / 21}$ and $h$ by $\sigma^{5 / 21}$ (see section 6.3.1); the lower inset shows the region around the first minima at $\zeta_{1}$, with $x$ scaled by $\sigma^{-7 / 63}$ and $h$ by $\sigma^{-5 / 63}$ (see section 6.3.2). The dashed line in the upper inset shows the asymptotic prediction for the first three bulges of a train given by (6.10), and in the lower inset shows the asymptotic prediction from (6.21). Panel (b) plots against $\sigma$ the maximum heights of the first two bulges, and the minimum height at their left hand borders. Pentagrams show the asymptotic predictions in (6.28) and (6.26); the maxima scale with $\sigma^{5 / 21}$, the minima with $\sigma^{-5 / 63}$.

subject to $h \rightarrow 1$ as $\zeta \rightarrow-\infty$, and the gap boundary conditions (3.6) at $\zeta=0$ (those conditions ensure continuity with the lag region in $\zeta>0$, and provide a total of three conditions on $h$ once the gap length $\ell$ is determined). Some solutions to this problem are shown in figure 10 for different values of $\sigma$.

\subsection{The effect of regularization on the shape of the travelling wave}

When the pre-wetted film is small, or the lag pressure is large, the boundary layer analysis of section 3.4 applies and indicates that

$$
h \rightarrow 0, \quad h^{\prime} \rightarrow 0, \quad h^{\prime \prime} \rightarrow \delta^{-1 / 5} \Gamma \text { or } \sigma^{1 / 7} \bar{\Gamma} \quad \text { for } \quad \zeta \rightarrow 0 .
$$

In this section, we consider the pre-wetted film with $\delta \ll 1$, for which the flux corrections on the right-hand side of (6.6) can be neglected, reducing that equation to (6.8). Thus, equivalent results for the fluid lag are obtained simply by replacing $\delta$ with $\sigma^{-5 / 7}$ and $\Gamma$ with $\bar{\Gamma}$.

\subsubsection{The primary bulge}

The relatively large second derivative arising in (6.9) implies that $h$ must become large directly behind the front, as seen in figures 9 and 10. This generates a wide bulge over which $h^{-2} \ll 1$ and the main balance in $(6.8)$ is $h^{\prime \prime \prime \prime \prime} \approx 1$. If the width of the bulge is $\zeta_{1}$, the height is therefore $h_{1} \sim O\left(\zeta_{1}^{5}\right)$. Moreover, given that the contact-line curvature is $h^{\prime \prime}=O\left(\delta^{-1 / 5}\right)$, the estimate $h^{\prime \prime} \sim h_{1} / \zeta_{1}^{2}$ indicates that the bulge has height $h_{1}=$ $O\left(\delta^{-1 / 3}\right)$, and length $\zeta_{1}=O\left(\delta^{-1 / 15}\right)$.

At the left-hand border of the bulge, $h$ must fall back to smaller values in order that the term $h^{-2}$ in (6.8) reenters the main balance and prevents the fluid depth from becoming

negative. This term then produces a sufficiently rapid adjustment of the fourth derivative 
of $h$ (i.e. the pressure) to cause $h$ to rebound and remain positive. As we demonstrate below, the transition layer in which $h$ rebounds turns out to be relatively narrow. As a result, the lower derivatives of $h$ remain largely unaffected by the structure of the transition region, which further demands that $h$ approaches the left edge of the bulge quadratically. Imposing the conditions $h=h^{\prime}=0$ at both borders of the bulge, we write the quintic solution for its shape as

$$
h=\frac{1}{120} \zeta^{2}\left(\zeta+\zeta_{1}\right)^{2}\left[\zeta+\zeta_{1}\left(\frac{1}{2}+\varphi_{1}\right)\right]
$$

where the contact-line curvature condition in (6.9) indicates that

$$
\zeta_{1}=\delta^{-1 / 15} Z_{1}, \quad Z_{1}=\left(120 \Gamma /\left(1+2 \varphi_{1}\right)\right)^{1 / 3},
$$

but the constant $\varphi_{1}$ is not yet determined. For use below, it is helpful to note that the non-zero derivatives at the right and left edges of the bulge are then

$$
h_{R / L}^{\prime \prime}=\delta^{-1 / 5} H_{R / L}^{\prime \prime}, \quad h_{R / L}^{\prime \prime \prime}=\delta^{-2 / 15} H_{R / L}^{\prime \prime \prime}, \quad h_{R / L}^{\prime \prime \prime \prime}=\delta^{-1 / 15} H_{R / L}^{\prime \prime \prime \prime},
$$

where

$$
H_{R / L}^{\prime \prime}=\frac{Z_{1}^{3}}{120}\left(2 \varphi_{1} \pm 1\right), \quad H_{R / L}^{\prime \prime \prime}=\frac{Z_{1}^{2}}{10}\left(1 \pm \varphi_{1}\right), \quad H_{R / L}^{\prime \prime \prime \prime}=\frac{Z_{1}}{10}\left(2 \varphi_{1} \pm 5\right) .
$$

\subsubsection{The minimum at the left border of the bulge}

The dominant balance in the transition region at the left-hand border of the bulge is $h^{\prime \prime \prime \prime \prime \prime} \sim-h^{-2}$, so that the jump in the fourth derivative across this region is

$$
-\left[h^{\prime \prime \prime \prime \prime}\right]_{\zeta_{1}^{-}}^{\zeta_{1}^{+}} \sim \int_{\zeta_{1}^{-}}^{\zeta_{1}^{+}} \frac{1}{h^{2}} \mathrm{~d} \zeta
$$

(the integral is taken over the width of the transition region, defined more precisely below). This is required from (6.12) to be of order $\delta^{-1 / 15}$, and the curvature of the transition region must also match the curvature of the bulge solution, $h^{\prime \prime}=O\left(\delta^{-1 / 5}\right)$. These balances indicate that the appropriate scaling of the transition region is

$$
\zeta=-\zeta_{1}+\delta^{7 / 45} \varsigma, \quad h=\delta^{1 / 9} \mathcal{G}(\varsigma)
$$

and the rescaled governing equation is therefore

$$
\mathcal{G}_{\varsigma \varsigma \varsigma \varsigma \varsigma}=-\frac{\delta^{4 / 9}}{\mathcal{G}^{2}}+\delta^{2 / 3} .
$$

Thus, to leading order, $\mathcal{G}$ is quartic and is determined simply by matching with the bulge solution, giving

$$
\mathcal{G} \sim G_{1}+\frac{1}{2} H_{L}^{\prime \prime} \varsigma^{2}+\frac{1}{6} \delta^{2 / 9} H_{L}^{\prime \prime \prime} \varsigma^{3}+O\left(\delta^{4 / 9}\right) .
$$

From this we deduce

$$
-\left[h^{\prime \prime \prime \prime}\right]_{\zeta_{1}^{-}}^{\zeta^{+}}=-\delta^{-23 / 45}\left[\mathcal{G}_{\varsigma \varsigma \varsigma \varsigma}\right]_{-\infty}^{\infty} \sim \int_{-\infty}^{\infty} \frac{\delta^{-1 / 15} d \varsigma}{\left(G_{1}+\frac{1}{2} H_{L}^{\prime \prime} \varsigma^{2}\right)^{2}}=\delta^{-1 / 15} \frac{2 \pi \sqrt{15}}{G_{1}^{3 / 2} Z_{1}^{3 / 2}\left(2 \varphi_{1}-1\right)^{1 / 2}} .
$$




\subsubsection{The wave train}

The solution for the transition region in (6.17) emphasizes how the second and third derivatives of $h(\zeta)$ are not modified to leading order by the local structure of this region. Thus, immediately to the left of the minimum at $\zeta=\zeta_{1}$, we must continue to integrate the travelling wave equation (6.8) subject to the conditions,

$$
h\left(\zeta_{1}^{+}\right)=0, \quad h^{\prime}\left(\zeta_{1}^{+}\right)=0, \quad h^{\prime \prime}\left(\zeta_{1}^{+}\right)=\delta^{-1 / 5} H_{L}^{\prime \prime} .
$$

But, save for the replace of $\Gamma$ by $H_{L}^{\prime \prime}$, these conditions are identical to those in (6.9), which kick off the primary bulge solution in (6.10). In other words, the minimum at $\zeta=\zeta_{1}$ must be followed by another bulge of width $\zeta_{2}=O\left(\delta^{-1 / 15}\right)$ and height $h_{2}=O\left(\delta^{-1 / 3}\right)$. Furthermore, that second bulge must terminate to the left in another transition region with the same structure as that elucidated in Sec. 6.3.2. Continuing the argument, we see that the unavoidable consequence is a train of successive bulges. Indeed, figure 10 presents numerical evidence for the development of a second bulge behind the primary one, scaling in the same asymptotic way, but algebraically smaller in size. Generalizing (6.10), the $k^{t h}$ bulge solution can be compactly written as

$$
h=\frac{1}{120}\left(\zeta+\sum_{j=1}^{k-1} \zeta_{j}\right)^{2}\left(\zeta+\sum_{j=1}^{k} \zeta_{j}\right)^{2}\left[\zeta+\sum_{j=1}^{k-1} \zeta_{j}+\zeta_{k}\left(\frac{1}{2}+\varphi_{k}\right)\right],
$$

which has derivatives at the edges given by (6.12) and (6.13) with subscript 1 replaced by $k$. The generalization of the leading order solution (6.17) for the $k^{\text {th }}$ transition region is similarly

$$
\mathcal{G}=G_{k}+\frac{Z_{k}^{3}}{240}\left(2 \varphi_{k}-1\right) \varsigma^{2}
$$

where $\zeta_{k}=\delta^{-1 / 15} Z_{k}$.

The final piece of the puzzle is to determine the constants, $\varphi_{k}$, and hence the values of $Z_{k}$ and $G_{k}$. In view of (6.21), matching of the second and third derivatives of $h$ across the $k^{\text {th }}$ transition region implies

$$
\lambda_{k} \equiv \frac{\zeta_{k+1}}{\zeta_{k}} \equiv \frac{Z_{k+1}}{Z_{k}}=\left(\frac{2 \varphi_{k}-1}{2 \varphi_{k+1}+1}\right)^{1 / 3}=\left(\frac{1-\varphi_{k}}{1+\varphi_{k+1}}\right)^{1 / 2} .
$$

This relation can be written as a cubic equation for $\varphi_{k+1}$, although one of its solutions, $\varphi_{k+1}=-\varphi_{k}$, is spurious. The remaining possible solutions are

$$
\varphi_{k+1}=\frac{1+\varphi_{k}-4 \varphi_{k}^{2} \pm\left(1-\varphi_{k}\right) \sqrt{5-8 \varphi_{k}}}{2\left(1-2 \varphi_{k}\right)^{2}} .
$$

These can be viewed as a two-fold mapping, $\varphi_{k+1}=F\left(\varphi_{k}\right)$, as illustrated in figure 11. However, none of the iterative solutions for $\varphi_{k}$ provides a sensible choice for these constants other than the fixed point,

$$
\varphi_{k} \equiv \varphi \approx 0.625 .
$$

Given this choice, we may complete our asymptotic solution by noting that the jump 


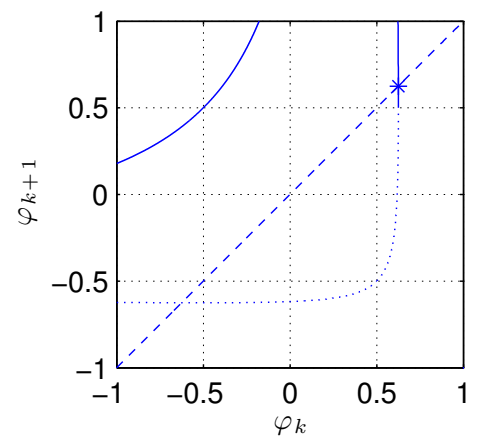

Figure 11. The two possible branches of the map $\varphi_{k+1}=F\left(\varphi_{k}\right)$ shown by solid and dotted lines (the upper branch diverges at $\varphi_{k}=0.5$ ). For $\varphi_{k}>\frac{5}{8}$, the solutions become complex and are not drawn. The dashed line is the diagonal, $\varphi_{k+1}=\varphi_{k}$ and the star indicates the fixed point, $\varphi_{k+1}=\varphi_{k}=\varphi$.

in the fourth derivative of $h$ across the $k^{t h}$ transition region must be

$$
-\left[h^{\prime \prime \prime \prime}\right]_{\zeta_{k}^{-}}^{\zeta^{+}}=\delta^{-1 / 15} Z_{k} \frac{(2 \varphi+5)(1-\varphi)^{1 / 2}-(2 \varphi-5)(1+\varphi)^{1 / 2}}{10(1+\varphi)^{1 / 2}} .
$$

Matching this with the $k^{\text {th }}$ generalization of (6.18) finally determines

$$
G_{k} \approx 17.33 Z_{k}^{-5 / 3} \text {. }
$$

In summary, in the limit $\delta \rightarrow 0$, the solution develops a train of bulges with widths, heights $h_{k} \propto \zeta_{k}^{5}$, and left-hand minima given by

$$
\zeta_{k}=\delta^{-1 / 15} Z_{k}, \quad h_{k}=\delta^{-1 / 3} H_{k}, \quad g_{k}=\delta^{1 / 9} G_{k},
$$

where

$$
Z_{k} \approx 3.76 \Gamma^{1 / 3} \lambda^{k-1}, \quad H_{k} \approx 0.26 \Gamma^{5 / 3} \lambda^{5(k-1)}, \quad G_{k} \approx 1.90 \Gamma^{-5 / 9} \lambda^{-5(k-1) / 3},
$$

and $\lambda \approx 0.48$ is the ratio of successive bulge widths. These predictions are compared with numerical solutions in figure 10. Note that the relatively small values of the exponents in (6.27), and the rapid decrease in successive bulge heights, signifies that very small values of $\delta$ and $\sigma^{-1}$ are required in order to observe the scaling of the second bulge. Note also that the train of waves does not continue forever. The bulge lengths $\zeta_{k}$ follow a geometric progression that sums to a finite value

$$
\sum_{k=1}^{\infty} \zeta_{k}=\delta^{-1 / 15} \frac{Z_{1}}{1-\lambda}
$$

and indeed the bulge heights reduce to become $O(1)$ when $k=O(\ln 1 / \delta)$. Thus the number of bulges in the train grows logarithmically with $\delta$ and one has to reduce $\delta$ (or equivalently increase $\sigma$ ) to quite extreme values even to see two of them in the numerical solutions. 

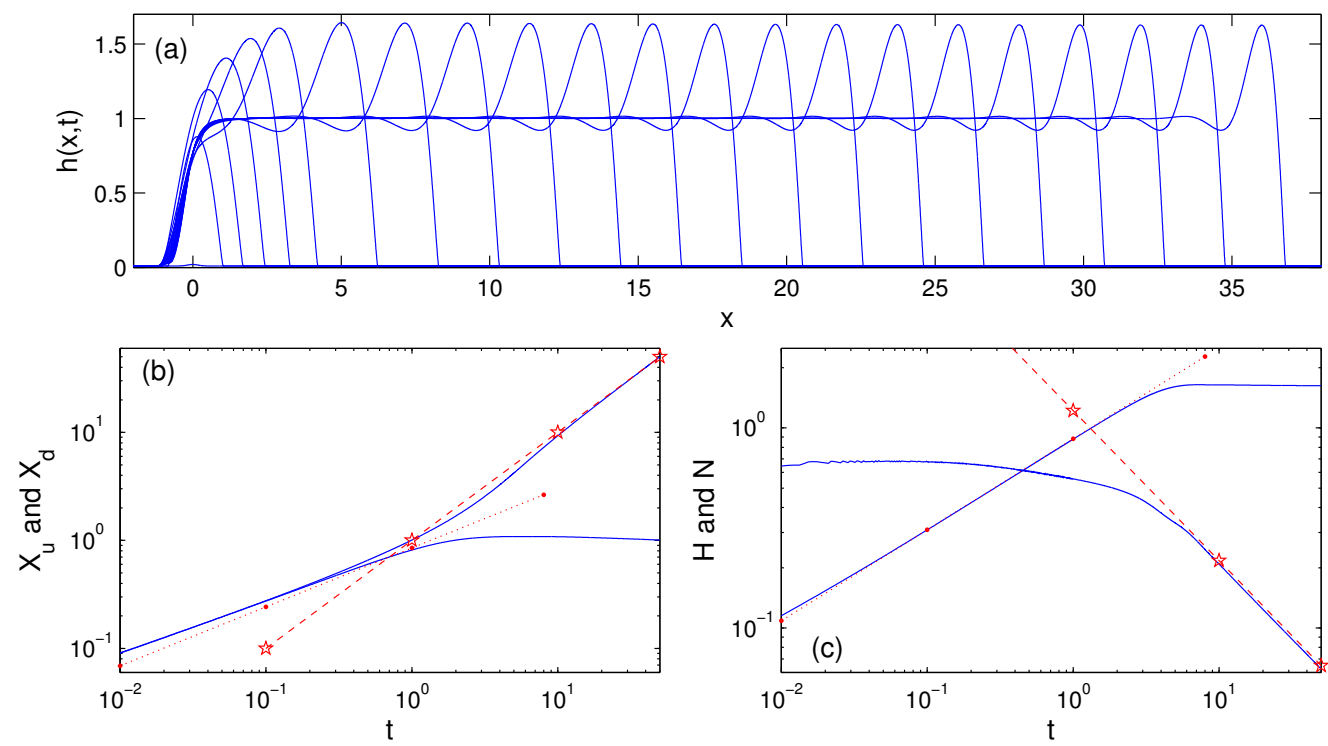

Figure 12. Numerical solutions for tension, slope and a pre-wetted film with $\delta=10^{-2}$. Panel (a) shows snapshots of $h$ at $t=1,2, \ldots, 5$, and then $7,9, \ldots, 37$. Panel (b) shows the evolution of the downstream and upstream fluid edges; the dotted line shows the early time solution from (5.8) and the dashed line shows the eventual linear trend of $X_{d}(t)$. Panel (c) shows the maximum height $H(t)$ and tension $N$; the dotted line show the early time behaviour from (5.8) and the dashed line shows the expected late-time dependence of $N$ from section 7.2.

\section{Sloping solutions with tension}

Finally, we consider a current on a slope, including tension but not bending: $\mathcal{B}=\mathcal{G}=0$ and $\mathcal{T}=\mathcal{S}=1$. The problem to be solved is

$$
h_{t}=\left[-h^{3}\left(N h_{x x x}+1\right)\right]_{x}+w, \quad N=\frac{1}{L_{u}+L_{d}} \int_{-L_{u}}^{L_{d}} \frac{1}{2} h_{x}^{2} \mathrm{~d} x .
$$

A numerical solution with a pre-wetted film is shown in figure 12. Aside from the nonconstant tension, this problem is equivalent to the standard problem of fluid flowing down a slope with surface tension (Tuck \& Schwarz 1990). The short time behaviour is the same as seen in section 5 . When the slope takes over, the bulk of the flow is dominated by the slope, with $h \approx 1$, and the tension comes into play only near the vent and downslope edge, where a prominent ridge appears. As the length of the fluid region expands, the tension decreases in time so that the effect of the tension on the shape of the front might also be expected to decay in time. The solution for the pre-wetted film in figure 12 suggests that the height of the ridge remains constant, however, while its length scale decreases in time. We confirm this observation below.

\subsection{Upstream spreading}

Around the vent, the tension allows the fluid depth to vary smoothly from $h \approx 1$ downstream to $h=0$ some distance upstream. However, the length scale over which this adjustment occurs is $O\left(N^{1 / 3}\right)$, which decays with $t$. Thus, once upstream spreading has 

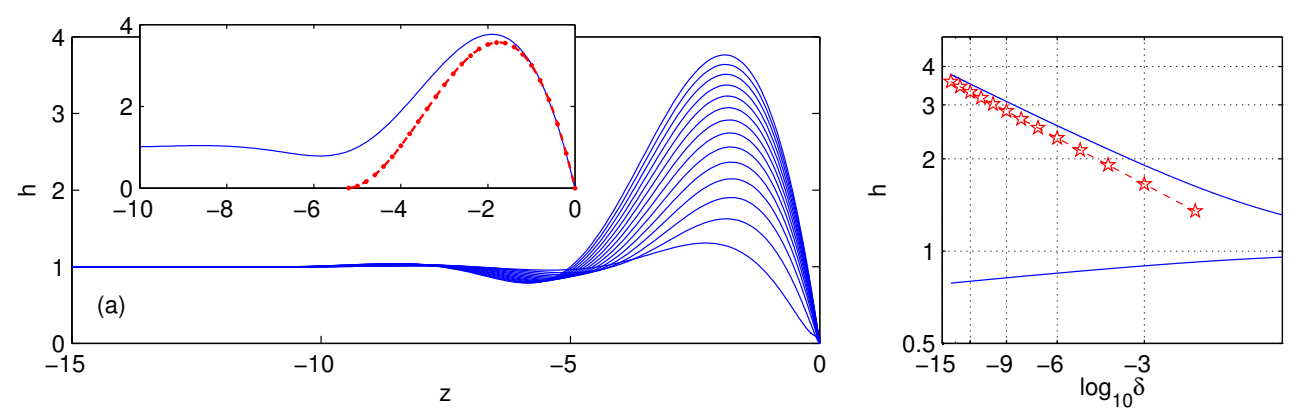

FIgURE 13. Numerical solutions for the travelling wave problem for tension and a pre-wetted film. Panel (a) shows the front profile for $\delta=10^{-1}, 10^{-2}, \ldots, 10^{-14}$; the inset compares the solution for $\delta=10^{-14}$ with the asymptotic result in (7.8). Panel (b) shows the maximum height of the first bulge, and the minimum height at its left edge, as $\delta$ is varied. Stars show the prediction from $(7.8)$, scaling with $(\ln 1 / \delta)^{1 / 2}$.

stopped and $X_{u}$ has reached its maximum value, the fluid subsequently drains back down the slope into this adjustment region and joins the main current.

At large times, the adjustment is quasi-steady and is largely confined to a region $x>-x_{*}(t)$ close to the vent, while an ever-thinning film occupies the interval $-X_{u}<$ $x<-x_{*}$ (the situation is similar to a fixed-volume drop draining on a slope; see [29]). As the draining film becomes very thin, one may effectively apply the boundary conditions $h=h_{x}=0$ at $-x_{*}$ (though we omit detailed justification here). Writing $x=N(t)^{1 / 3} z$ and $x_{*}(t)=N(t)^{1 / 3} z_{*}$, we see that the shape of the adjustment region is described by

$$
-h_{z z z}= \begin{cases}1 & -z_{*}<z<0 \\ 1-1 / h^{3} & 0<z,\end{cases}
$$

with boundary conditions $h=h_{z}=0$ at $z=-z_{*}$ and $h \rightarrow 1$ as $z \rightarrow \infty$. Numerical solution of this problem determines $z_{*} \approx 1.73$.

\subsection{The travelling wave}

Considering first the case of the pre-wetted film, we again study the travelling wave by working in the translating frame of the front. This time the relevant length scale depends on the tension $N(t)$ : we write

$$
h=h(\zeta), \quad \zeta=N^{-1 / 3}\left(x-X_{d}\right),
$$

and neglect the time derivative in (7.1) (which can be justified since $N$ decays with $t$ ) to furnish

$$
-\dot{X}_{d} h^{\prime}=\left[-h^{3}\left(h^{\prime \prime \prime}+1\right)\right]^{\prime},
$$

with $h \rightarrow 1$ as $\zeta \rightarrow-\infty$ and $h \rightarrow \delta$ as $\zeta \rightarrow \infty$. Solutions to this problem are shown in figure 13 .

The fact that this rescaled problem becomes independent of the tension rationalizes the observation that the height of the travelling wave remains constant in time. Moreover, contributions to the tension arise only from the vicinity of the vent and the travelling 
front, so the integral for the tension becomes

$$
N \sim \frac{1}{N^{1 / 3} t}\left[\int_{-z_{*}}^{\infty} \frac{1}{2} h_{z}^{2} \mathrm{~d} z+\int_{-\infty}^{\infty} \frac{1}{2} h^{\prime 2} \mathrm{~d} \zeta\right]
$$

$\left(L_{u}+L_{d} \sim t\right)$. Thus, $N \sim B t^{-3 / 4}$, where the constant $B$ depends on $\delta$ in view of the boundary condition for (7.4). The characteristic width of the bulge at the front therefore decreases in time as $t^{-1 / 4}$. For $\delta=10^{-2}, B \approx 1.22$ and the asymptotic prediction for $N(t)$ is included in figure 12. ${ }^{1}$

For the fluid lag the situation is less clear-cut: the boundary conditions (3.8) apply at $\zeta=0$, and indicate that the travelling wave solution depends on a time-dependent regularization parameter, $\hat{\sigma}=\sigma N^{-1 / 3}$. However, at least when $N \gg \sigma^{-1}$, the effective boundary conditions in (3.14) hold and the travelling wave shape for the fluid lag becomes equivalent to that for the pre-wetted film.

\subsection{The effect of regularization on the shape of the travelling wave}

For $\delta \ll 1$, the integral of (7.4) furnishes

$$
h^{\prime \prime \prime}=-1+h^{-2},
$$

ignoring the flux corrections in the pre-wetted film. We apply $h \rightarrow 1$ for $\zeta \rightarrow-\infty$, and the effective contact angle conditions in (3.14),

$$
h=0, \quad h^{\prime}=-3^{1 / 3}(\ln 1 / \delta)^{1 / 3} \quad \text { at } \quad \zeta=0 .
$$

The asymptotic structure of the solution to this problem was analyzed by Benilov et al [2], and consists of a train of bulges in which the dominant balance is $h^{\prime \prime \prime} \approx-1$. The first bulge has the shape

$$
h=-\frac{1}{6} \zeta\left(\zeta+\zeta_{1}\right)^{2}, \quad \zeta_{1}=(\ln 1 / \delta)^{1 / 6} 2^{1 / 2} 3^{2 / 3},
$$

with a maximum height of $h_{1}=(\ln 1 / \delta)^{1 / 2} 2^{5 / 2} / 9$. As seen in figure 13 , extremely small values of $\delta$ are required to make this approximation remotely close to the full solution, the error in the predicted maximum height for $\delta=10^{-15}$ still being around $5 \%$.

Unlike in the travelling front problem for bending, when the amplitude of successive bulges decayed algebraically, the bulges in this case have asymptotically lower amplitude, each bulge being logarithmically smaller than the previous one. There is little hope of being able to distinguish this in numerical solutions with finite values of $\delta$ or $\sigma$. Similar asymptotically decaying trains of capillary waves also occur in other settings $[31,8]$.

1 This solution for the tension will become invalid once the length of the current is sufficiently large that the fluid traction acting over its great length causes a significant gradient in tension. As noted in section 2, this has been ignored from our analysis, but with the adopted nondimensionalization the traction per unit length exerted by the uniform current would be $\frac{1}{2} \varepsilon^{2}$, where $\varepsilon=\mathcal{H} / \mathcal{L}$ is the characteristic aspect ratio, and the longitudinal force balance is therefore $\partial N / \partial x=-\frac{1}{2} \varepsilon^{2}$. It can be shown that this will eventually lead to an increase in the average tension once $X_{d} \sim t \sim O\left(\varepsilon^{-8 / 7}\right.$ ) (the tension at the downstream end continues to decrease towards zero however). A similar modification would apply to the results in section 5 once the current is so large that traction on the sheet becomes important. 


\section{Discussion}

We have analyzed solutions of a higher-order nonlinear diffusion equation for a fluid spreading underneath an elastic sheet. Our primary focus has been on the behaviour close to the advancing fronts at the edges of the fluid and the role this has in controlling the dynamics. The early time behaviour always depends on the detailed dynamics of those contact lines. We have used two regularizations of the contact region to explore this dependence: a pre-wetted film and a fluid lag. For long times, the spreading is always eventually controlled by gravity, although the time taken to reach this state depends upon the choice of the parameters inherent in the regularization (here, the film thickness, $\delta$, or the lag pressure, $\sigma$ ). More extreme values of these parameters have the effect of confining the flow to a narrower region and lengthening the time taken for gravity to take control.

Our analysis of tension-dominated spreading demonstrates that solutions are qualitatively similar to those for a current restrained by surface tension; the decay of the elastic tension in time mostly alters how the length and depth of the current scale with time, and cause any boundary layers in which the tension controls the dynamics to gradually thin. With bending, the dependence of the solutions on the regularization parameters at the edge is stronger, depending on a weak power rather than a logarithm (compare figures 5,6 and 8). In the case of the travelling wave on the slope, the bending stress creates a decaying wave train extending behind the front; this feature has some similarities with the train of capillary waves seen in the corresponding surface tension problem [2] (and, indeed, the problem with elastic tension), although the asymptotic structure is somewhat different and more readily observable.

For tension-dominated problems, the solutions depend logarithmically on the regularization parameters. The origin of this dependence is the match between the three powers of the fluid depth $h$ in the effective diffusion coefficient and the three derivatives of $h$ in the pressure gradient. This corresponds to a critical situation in a wider class of such equations with other exponents [9]. Further work could extend our analysis to other values of the exponent of $h$ in the diffusivity; that such choices may lead to different behaviour is physically important because other exponents emerge in alternative physical models, such as for flow in porous media. The difficulties associated with the degeneracy of the equation at the edges are in part due to the approximation of the elastic stresses using the beam equation (2.2); another relevant extension would be to reintroduce the full elastic equations for the sheet close to the contact line and impose an appropriate condition on the stress singularity at the fracture-like tip [20,4].

We have focussed on planar spreading as the simplest context in which to understand the behaviour at the fluid edge. Most applicable settings are two dimensional, however, in which case the tension becomes spatially varying as well as time-dependent (e.g. [5]). Even without that extra layer of complexity, the direct extension of our numerical solutions to two dimensions (unless axisymmetric) is problematic, because a fine resolution is required to resolve the detailed structure of the region near the contact "contour". A potential use for an analysis of the type presented here is to formulate effective boundary conditions that avoid the need for such detailed resolution. Alternatively, one could explore the stability of the planar travelling front solutions towards transverse perturbations; indeed 
it is highly likely that the fronts suffer a fingering instability as in the corresponding surface tension problem [3, 6]. A step in this direction has been taken by Khomenko [17].

For clarity, we have separately addressed the cases when bending and tension provide the stress from the elastic sheet. In general, however, both terms appear in the equation and one may wonder how this may affect the general features of the spreading dynamics. Generically, one expects that the higher derivatives of the bending stress impact the problem more at earlier times than the tension, which becomes more prominent later in the evolution, before gravity eventually wins out. Thus, solutions may experience a variety of different evolutionary phases due to the interplay of bending, tension and gravity. Provided the bending term is present, that term will always dominate the behaviour in the narrow region adjoining the contact line, and the asymptotic solutions that we have presented can be modified to account for situations with a combination of bending, tension and gravity controlling the pressure over the fluid interior. A recent study [22] has considered the axisymmetric analogue of this problem, and explored such solutions with a tension-dominated interior matching to a bending-dominated edge region.

\section{Acknowledgements}

IJH acknowledges the Killam Foundation and NSERC for the support of a postdoctoral fellowship. We thank L. Mahadevan, J. McElwaine and C. Schoof for useful discussions, and particularly thank the anonymous reviewers for their careful scrutiny of the manuscript.

\section{Appendix A Boundary layers at the fluid front}

\section{A.1 Pre-wetted film with bending}

When $\mathcal{B} \neq 0$, bending dominates the pressure gradient on small length scales. Moreover, for a pre-wetted film, $h \rightarrow \delta$ ahead of the fluid front. Thus, we take

$$
\epsilon=\delta^{3 / 5} \mathcal{B}^{1 / 5} \dot{X}^{-1 / 5} \quad \text { and } \quad \Delta=\delta
$$

in (3.9). The integral of (3.11a) leads to

$$
1-g=g^{3} g_{\xi \xi \xi \xi \xi} \quad \text { with } \quad g \rightarrow 1 \quad \text { as } \quad \xi \rightarrow \infty .
$$

In order to match to the bulk of the fluid flow, we require $g(\xi)$ to grow at most quadratically as $\xi \rightarrow-\infty$ :

$$
g \sim \frac{1}{2} \Gamma \xi^{2} \quad \text { as } \quad \xi \rightarrow-\infty .
$$

The numerical solution to (A 2), which is shown in figure 2 , provides $\Gamma \approx 1.35$. In terms of the original variables, the limiting behaviour in (A 3) furnishes the first relation in (3.13).

\section{A.2 Fluid lag with bending}

For a fluid lag, the pressure condition $\mathcal{B} h_{x x x x}=-\sigma$ at $x=X$ implies

$$
\varepsilon=\sigma^{-3 / 7} \mathcal{B}^{2 / 7} \dot{X}^{1 / 7} \quad \text { and } \quad \Delta=\sigma^{-5 / 7} \mathcal{B}^{1 / 7} \dot{X}^{4 / 7} .
$$


The fluid occupies $\xi<0$ and the jump condition (3.1) applies at $\xi=0$, as do the four conditions in (3.6) that describe the lag region. The leading order boundary layer problem is therefore

$$
-1=g^{2} g_{\xi \xi \xi \xi \xi}
$$

with

$$
72 g+24 \Lambda g_{\xi}-\Lambda^{4}=24 g-4 \Lambda^{2} g_{\xi \xi}-\Lambda^{4}=24 g+4 \Lambda^{3} g_{\xi \xi \xi}-3 \Lambda^{4}=g_{\xi \xi \xi \xi}+1=0
$$

at $\xi=0$. Here $\Lambda=\sigma^{3 / 7} \mathcal{B}^{-2 / 7} \dot{X}^{-1 / 7} \ell$ is the rescaled lag, which is determined as part of the solution.

Numerical solution now gives

$$
g \rightarrow \frac{1}{2} \bar{\Gamma} \xi^{2} \quad \text { as } \quad \xi \rightarrow-\infty,
$$

where $\bar{\Gamma} \approx 1.77$, and the scaled lag is $\Lambda \approx 1.33$.

\section{A.3 Pre-wetted film with tension}

If there is no bending term, $\mathcal{B}=0$, and the tension dominates the pressure gradient near the front. The local problem at the front is analogous to that with surface tension $[30,28]$. We write

$$
\varepsilon=\delta \mathcal{T}^{1 / 3} N^{1 / 3} \dot{X}^{-1 / 3} \quad \text { and } \quad \Delta=\delta,
$$

and the integral of $(3.11 \mathrm{~b})$ provides

$$
g-1=g^{3} g_{\xi \xi \xi}, \quad \text { with } \quad g \rightarrow 1 \quad \text { as } \quad \xi \rightarrow \infty .
$$

Matching now demands the limiting behaviour,

$$
g \sim-3^{1 / 3} \xi(\ln (-\xi))^{1 / 3} \text { as } \xi \rightarrow-\infty .
$$

Or, in terms of the original variables,

$$
h \sim 3^{1 / 3}\left[\ln \left(\delta^{-1} \mathcal{T}^{-1 / 3} N^{-1 / 3} \dot{X}^{1 / 3}(X-x)\right)\right]^{1 / 3} \mathcal{T}^{-1 / 3} N^{-1 / 3} \dot{X}^{1 / 3}(X-x) .
$$

In translating this relation to the final condition in (3.14), we neglect the terms $\dot{X}$ and $N$ in the logarithm, as well as the dependence on $x$. This assumes implicitly that these quantities are much larger than $\delta$ (or $\sigma^{-1}$ below), which is asymptotically correct for small enough $t$. However $\dot{X}$ and $N$ decay in time and such an approximation is strictly invalid once $\ln t=O(\ln 1 / \delta)($ or $O(\ln \sigma))$. We avoid explicit consideration of such large times in the current exploration.

\section{A.4 Fluid lag with tension}

In this case, the condition $\mathcal{T} N h_{x x}=\sigma$ at $x=X$ indicates that

$$
\varepsilon=\sigma^{-1} \mathcal{T}^{2 / 3} N^{2 / 3} \dot{X}^{1 / 3} \quad \text { and } \quad \Delta=\sigma^{-1} \mathcal{T}^{1 / 3} N^{1 / 3} \dot{X}^{2 / 3} .
$$

The integral of $(3.11 b)$ is

$$
1=g^{2} g_{\xi \xi \xi}
$$


with the boundary conditions (3.8) becoming

$$
g-\frac{1}{2} \Lambda^{2}=g_{\xi}+\Lambda=g_{\xi \xi}-1=0 \quad \text { at } \quad \xi=0,
$$

where $\Lambda=\sigma \mathcal{T}^{-2 / 3} N^{-2 / 3} \dot{X}^{-1 / 3} \ell$ is the rescaled lag. Again,

$$
g \sim-3^{1 / 3} \xi(\ln (-\xi))^{1 / 3} \quad \text { as } \quad \xi \rightarrow-\infty,
$$

or

$$
h \sim 3^{1 / 3}\left[\ln \left(\sigma \mathcal{T}^{-2 / 3} N^{-2 / 3} \dot{X}^{-1 / 3}(X-x)\right)\right]^{1 / 3} \mathcal{T}^{-1 / 3} N^{-1 / 3} \dot{X}^{1 / 3}(X-x) .
$$

\section{References}

[1] Aristoff, J.M., Duprat, C., Stone, H.A. 2011 Elastocapillary imbibition Int. J. Non-Linear Mech. 46, 648-656

[2] Benilov, E.S., Chapman, S.J., McLeod, J.B., Ockendon, J.R., Zubkov, V.S 2010 On liquid films on an inclined plane J. Fluid Mech. 663,53-69

[3] Bonn, D., Eggers, J., Indekeu, J., Meunier, J., Rolley, E. 2009 Wetting and spreading Rev. Modern Phys. 81,739-805

[4] Bunger. A.P., Detournay, E. 2005 Asymptotic solution for a penny-shaped near-surface hydraulic fracture Engin. Fracture Mech. 72, 2468-2486

[5] Chopin, J., Vella, D., Boudaoud, A. 2008 The liquid blister test Proc. R. Soc. A 464,28872906

[6] Craster, R.V. and Matar, O.K. 2009 Dynamics and stability of thin liquid films. Rev. Modern Phys. 81,1131-1198

[7] Das, S.B, Joughin, I., Behn, M., Howat, I., King, M.A., Lizarralde, D., Bhatia, M.P. 2008 Fracture propagation to the base of the Greenland ice sheet during supraglacial lake drainage Science 320,778-781

[8] Duchemin, L., Lister, J.R., Lange, U. 2005 Static shapes of levitated viscous drops. J. Fluid Mech. 533,161-170

[9] Flitton, J.C. and King, J.R. 2004 Moving-boundary and fixed-domain problems for a sixthorder thin-film equation. Euro. J. Appl. Math. 15,713-754

[10] Garagash, D., Detournay, E. 2000 The tip region of a fluid-driven fracture in an elastic medium. J. Appl. Mech. 67,183-192

[11] Gaver, D.P., Halpern, D., Jensen, O.E., Grotberg, J.B. 1996 The steady motion of a semiinfinite bubble through a flexible-walled channel. J. Fluid Mech. 319,25-65

[12] Gordeliy, E., Detournay, E. 2011 A fixed grid algorithm for simulating the propagation of a shallow hydraulic fracture with a fluid lag. Int. J. Numeric. Anal. Meth. Geomech. $\mathbf{3 5}, 602-629$

[13] Heil, M., Hazel, A. 2011 Fluid-structure interaction in internal physiological flows. Ann. Rev. Fluid Mech. 43,141-162

[14] Hosoi, A.E., Mahadevan, L. 2004 Peeling, healing and bursting in a lubricated elastic sheet Phys. Rev. Lett. 93, 137802

[15] Huppert, H.E. 1982 The propagation of two-dimensional and axisymmetric viscous gravity currents over a rigid horizontal surface. J. Fluid Mech. 121,43-58

[16] Huang, R., Suo, Z. 2002 Wrinkling of a compressed elastic film on a viscous layer. J. Appl. Phys. 91,1135-1142

[17] Khomenko, M. 2010 Viscous fluid instabilities under an elastic sheet. MSc. Thesis, University of British Columbia

[18] King, J.R. and Bowen, M. 2001 Moving boundary problems and non-uniqueness for the thin film equation. Euro J. Appl. Math. 12,321-356

[19] King, J. R. 1989 The isolation oxidation of silicon the reaction-controlled case. SIAM J. Appl. Math. 49,1064-1080. 
[20] Lister, J.R. 1990 Buoyancy-driven fluid fracture : the effects of material toughness and of low-viscosity precursors. J. Fluid Mech. 210,263-280

[21] Lister, J.R. 1992 Viscous flows down an inclined plane from point and line sources. J. Fluid Mech. 242,631-653

[22] Lister, J.R., Peng, G. G., Neufeld, J.Viscous control of peeling an elastic sheet by bending and pulling. Phys. Rev. Lett. 111, 154501.

[23] Michaut, C. 2011 Dynamics of magmatic intrusions in the upper crust: Theory and applications to laccoliths on Earth and the Moon. J. Geophys. Res. 116,doi:10.1029/2010JB008108

[24] Myers, T.G. 1998 Thin films with high surface tension. SIAM Review 40,441-462

[25] Pihler-Puzović, D., Illien, P., Heil, M., Juel, A. Suppression of complex fingerlike patterns at the interface between air and a viscous fluid by elastic membranes. Phys. Rev. Lett. 108,074502

[26] Tanner, L.H. 1979 The spreading of silicone oil drops on horizontal surfaces J. Phys. D: Appl. Phys. 12,1473-1484

[27] Tsai, V.C., Rice, J.R. 2012 Modeling turbulent hydraulic fracture near a free surface J. App. Mech. 79,031003

[28] Tuck, E.O., Schwartz, L.W. 1990 A numerical and asymptotic study of some third-order ordinary differential equations relevant to draining and coating flows. SIAM Review $\mathbf{3 2}$, 453-469

[29] Tuck, E.O., Schwartz, L.W. 1991 Thin static drops with a free attachment boundary J. Fluid Mech. 223, 313-324

[30] Voinov, O.V. 1977 Inclination angles of the boundary in moving liquid layers. J. Appl. Mech. Tech. Phys. 18,216-222

[31] Wilson, S.D.R., Jones, A.F. 1983 The entry of a falling film into a pool and the airentrainment problem J. Fluid Mech. 128,219-230 